\title{
TANGENTIAL ALEXANDER POLYNOMIALS AND NON-REDUCED DEGENERATION
}

\author{
MUTSUO OKA
}

\begin{abstract}
We introduce a notion of tangential Alexander polynomials for plane curves and study the relation with $\theta$-Alexander polynomial. As an application, we use these polynomials to study a non-reduced degeneration $C_{t}, \rightarrow D_{0}+j L$. We show that there exists a certain surjectivity of the fundamental groups and divisibility among their Alexander polynomials.
\end{abstract}

\section{INTRODUCTION}

Let $C$ be a plane curve. We are interested in the geometry of plane curves. Choose a line $L \subset \mathbb{P}^{2}$ and put $\mathbb{C}_{L}^{2}:=\mathbb{P}^{2}-L$. As geometrical invariants, we consider

(a) Fundamental groups: $\pi_{1}\left(\mathbb{P}^{2}-C\right)$ and $\pi_{1}\left(\mathbb{C}_{L}^{2}-C\right)$

(b) Alexander polynomial $\Delta_{C}(t ; L)$.

Zariski studied $\pi_{1}\left(\mathbb{P}^{2}-C\right)$ systematically [36] and further developments have been made by many authors. To compute the Alexander polynomial, we need to choose a line at infinity $L$. However for a generic $L$, the Alexander polynomial has too much restrictions and we have often the trivial case $\Delta_{C}(t ; L)=(t-1)^{r-1}$ where $r$ is the number of the irreducible components. In our previous paper 24], we have introduced the notion of $\theta$-Alexander polynomials. This gives more informations for certain reducible curves but it does not give any further information for irreducible curves.

The purpose of this paper is to introduce the notion of the tangential Alexander polynomials. Namely we consider all tangent lines $T_{P} C$ for the line at infinity. It turns out that tangential Alexander polynomials are related to $\theta$-Alexander polynomials. We apply these polynomials to study non-reduced degenerations. Let $C_{t}, t \in \Delta$ be a analytic family of reduced curves for $t \neq 0$ such that $C_{t} \stackrel{t \rightarrow \infty}{\longrightarrow} C_{0}=D_{0}+j L$ where $L$ is a line. The case $j \geq 2$ is a typical non-reduced degeneration. In this situation we study the geometry of $D_{0}$ using that of $C_{t}$. One of our results is the surjectivity assertion of the natural homomorphism:

$$
\phi: \pi_{1}\left(\mathbb{C}_{L}^{2}-D_{0}\right) \rightarrow \pi_{1}\left(\mathbb{C}_{L}^{2}-C_{t}\right)
$$

Here the point is that $L$ is the line component of the limit curve $C_{0}$ ( Theorem 14 $\S 5$ ). This paper consists of the following sections.

$\S 2$ Fundamental groups

$\S 3$ Alexander polynomial

$\S 4$ Dual stratification and tangential fundamental groups

$\S 5$ Degeneration into non-reduced curves with a multiple line

1991 Mathematics Subject Classification. 14H30,14H45, 32S55.

Key words and phrases. tangential Alexander polynomial, non-reduced degeneration. 


\section{Fundamental groups}

Let $L$ be a fixed line and put $\mathbb{C}_{L}^{2}:=\mathbb{P}^{2}-L$. We say $L$ is generic with respect to $C$ if $L$ and $C$ intersects transversely. The topology of $\mathbb{C}_{L}^{2}-C$ does not depend on $L$ if $L$ is generic and we call it the generic affine complement and we often write as $\mathbb{C}^{2}$ instead of $\mathbb{C}_{L}^{2}$. The following Lemma describes the relation of two fundamental groups.

Lemma 1. ([17]) Let $\omega$ be a lasso for $L$ and $N(\omega)$ be the subgroup normally generated by $\omega$. (1) The following sequence is exact.

$$
1 \rightarrow \mathbf{N}(\omega) \rightarrow \pi_{1}\left(\mathbb{C}_{L}^{2}-C, b_{0}\right) \rightarrow \pi_{1}\left(\mathbb{P}^{2}-C, b_{0}\right) \rightarrow 1
$$

(2) Assume that $L$ is generic. Then

(i) $\omega$ is in the center of $\pi_{1}\left(\mathbb{C}_{L}^{2}-C\right)$ and $\mathbf{N}(\omega) \cong \mathbb{Z}$.

(ii) We have the equality $D\left(\pi_{1}\left(\mathbb{C}^{2}-C\right)\right)=D\left(\pi_{1}\left(\mathbb{P}^{2}-C\right)\right)$ among their commutator groups. Thus $\pi_{1}\left(\mathbb{P}^{2}-C\right)$ is abelian if and only if $\pi_{1}\left(\mathbb{C}^{2}-C\right)$ is abelian.

For non-generic line $L, \pi_{1}\left(\mathbb{C}_{L}^{2}-C\right)$ may be non-abelian even if $\pi_{1}\left(\mathbb{P}^{2}-C\right)$ is abelian. For example, let $C=\left\{Y^{2} Z-X^{3}=0\right\}$ and take $L=\{Z=0\}$. Then $\pi_{1}\left(\mathbb{C}_{L}^{2}-C\right) \cong B_{3}$ where $B_{3}$ is the braid group of three strings and we recall that $B_{3} \cong\langle a, b \mid a b a=b a b\rangle$ ([4]).

2.1. First homology group $H_{1}\left(\mathbb{P}^{2}-C\right)$. Assume that $C$ is a projective curve with $r$ irreducible components $C_{1}, \ldots, C_{r}$ of degree $d_{1}, \ldots, d_{r}$ respectively. By Lefschetz duality, we have the following.

Proposition 2. $H_{1}\left(\mathbb{P}^{2}-C, \mathbb{Z}\right)$ is isomorphic to $\mathbb{Z}^{r-1} \times\left(\mathbb{Z} / d_{0} \mathbb{Z}\right)$ where $d_{0}=\operatorname{gcd}\left(d_{1}, \ldots, d_{r}\right)$. In particular, $H_{1}\left(\mathbb{C}_{L}^{2}-C\right) \cong \mathbb{Z}^{r}$.

Take a lasso $g_{i}$ for each component $C_{i}$ of $C$ for $i=1, \ldots, r$. Then the corresponding homology classes $\left\{\left[g_{i}\right], i=1, \ldots, r\right\}$ give free abelian generators of $H_{1}\left(\mathbb{C}_{L}^{2}-C\right)$.

2.2. Degenerations and fundamental groups. Let $C$ be a reduced plane curve. The total Milnor number $\mu(C)$ is defined by the sum of the local Milnor numbers $\mu(C, P)$ at the singular points $P$ of $C$. Let $\Delta:=\{\zeta \in \mathbb{C}|| \zeta \mid \leq 1\}$ the unit disk. We consider an analytic family of projective curves $C_{t}=\left\{F_{t}(X, Y, Z)=0\right\}, t \in \Delta$ where $F_{t}(X, Y, Z)$ are reduced homogeneous polynomial of degree $d$ for any $t$. We call $\left\{C_{t} ; t \in \Delta\right\}$ a reduced degeneration. We assume that $C_{t}, t \neq 0$ have the same configuration of singularities so that they are topologically equivalent but $C_{0}$ may obtain more singularities, i.e., $\mu\left(C_{t}\right) \leq \mu\left(C_{0}\right)$.

Theorem 3. For a given reduced degeneration $\left\{C_{t} ; t \in \Delta\right\}$, there is a canonical surjective homomorphism for $t \neq 0$ :

$$
\varphi: \pi_{1}\left(\mathbb{P}^{2}-C_{0}\right) \rightarrow \pi_{1}\left(\mathbb{P}^{2}-C_{t}\right)
$$

In particular, if $\pi_{1}\left(\mathbb{P}^{2}-C_{0}\right)$ is abelian, so is $\pi_{1}\left(\mathbb{P}^{2}-C_{t}\right)$.

See for example, 24] and also Theorem [14 of $\S 5$ for another simple proof. 
2.3. Product formula. Assume that $C_{i}$ is a curve of degree $d_{i}, i=1,2$ which are intersecting transversely at $d_{1} d_{2}$ distinct points. We denote the transversality as $C_{1} \pitchfork C_{2}$. Take a line $L$ such that $L \cap C_{1} \cap C_{2}=\emptyset$. Note that $L$ need not be generic for $C_{1}$ or $C_{2}$.

Theorem 4. (Oka-Sakamoto [28]) Under the above assumption, we have

$$
\pi_{1}\left(\mathbb{C}_{L}^{2}-C_{1} \cup C_{2}\right) \cong \pi_{1}\left(\mathbb{C}_{L}^{2}-C_{1}\right) \times \pi_{1}\left(\mathbb{C}_{L}^{2}-C_{2}\right)
$$

For further information about fundamental groups, we refer to [4, 12, 18, 20, 32].

\subsection{Example.}

2.4.1. Abelian cases. A curve $C$ with small singularities has often commutative fundamental group $\pi_{1}\left(\mathbb{P}^{2}-C\right)$. Some examples are here:

$-C$ is a smooth irreducible curve.

- Irreducible curves with only $A_{1}$-singularities (i.e., nodes) by [36, 9, 8, 10, 16, 29, or irreducible curve of degree $d$ with $a$ nodes and $b$ cusps (i.e., $A_{2}$ ) with $6 b+2 a<d^{2}$ ([16]).

$-\pi_{1}\left(\mathbb{P}^{2}-C\right)$ (respectively $\pi_{1}\left(\mathbb{C}_{L}^{2}-C\right)$ ) is abelian for any irreducible curve of degree $d$ if it has a flex of order $\geq d-3$ in $\mathbb{C}_{L}^{2}$ (resp. of order $\left.d-2\right)([36])$.

Let $f: \mathbb{C}^{2} \rightarrow \mathbb{C}$ be a polynomial mapping. Recall that $\alpha$ is a atypical value at infinity if the topological triviality at infinity fails at $t=\alpha$ for the family of curves $C_{t}:=\overline{f^{-1}(t)}$ (see [34).

Proposition 5. (20]) Let $f: \mathbb{C}^{2} \rightarrow \mathbb{C}$ be a polynomial mapping and assume that 0 is not an atypical value at infinity and $C=f^{-1}(0)$ is smooth in $\mathbb{C}^{2}$. Then $\pi_{1}\left(\mathbb{C}^{2}-C\right) \cong \mathbb{Z}$.

2.4.2. Non-abelian case. Assume that $p, q$ are positive integers greater than 1 and consider the curve

$$
C_{p, q}: \quad f_{p}(X, Y, Z)^{q}+f_{q}(X, Y, Z)^{p}=0
$$

where $f_{p}, f_{q}$ are polynomials of degree $p, q$ respectively. $C_{p, q}$ is called a curve of $(p, q)$-torus type. Assume that two curves $\left\{f_{p}=0\right\}$ and $\left\{f_{q}=0\right\}$ intersect transversely and there is no other singularities of $C_{p . q}$. Then $\pi_{1}\left(\mathbb{P}^{2}-C_{p, q}\right) \cong G(p, q, q)$ and $\pi_{1}\left(\mathbb{C}^{2}-C\right) \cong G(p, q)$. In particular, if $p, q$ are coprime, $\pi_{1}\left(\mathbb{P}^{2}-C_{p, q}\right) \cong \mathbb{Z}_{p} * \mathbb{Z}_{q}$. For the definition of $G(p, q)$ and $G(p, q, r)$, we refer to $[18$.

2.5. Class formula and flex formula. For the study of curves of low degree, it is often important to know the existence of flex points. Let $d=$ degree $(C), \breve{d}$ be the degree of the dual curve $\check{C}$, let $\Sigma(C)$ be the singular points of $C$ and let $\alpha(C)$ be the number of the flex points. Then $\breve{d}$ and $\alpha(C)$ are given by the formula:

$$
\begin{gathered}
\check{d}=d(d-1)-\sum_{P \in \Sigma(C)}(\mu(C, P)+m(C, P)-1) \\
\alpha(C)=3 d(d-2)-\sum_{P \in \Sigma(C)} \gamma(C, P)
\end{gathered}
$$

where $m(C, p)$ is the multiplicity of $C$ at $P$ and $\gamma(C, P)$ is the flex defect of the singularity $(C, P)$ [15, 21]. (In 21], we have denoted $\gamma(C, P)$ as $\delta(C, P)$. To distinguish with $\delta$-genus of the singularity, we change the notation.) 


\section{Alexander polynomial}

3.1. General definition. Let $X$ be a finite connected CW-complex and let $\phi: \pi_{1}(X) \rightarrow \mathbb{Z}$ be a surjective homomorphism. We fix a generator $t$ of the infinite cyclic group $\mathbb{Z}$. Let $\Lambda$ is the group ring of $\mathbb{Z}$. Then $\Lambda$ is isomorphic to the Laurent polynomial ring $\mathbb{C}\left[t, t^{-1}\right]$ and $\Lambda$ is a principal ideal domain. Consider an infinite cyclic covering $p: \widetilde{X} \rightarrow X$ such that $p_{\#}\left(\pi_{1}(\tilde{X})\right)=\operatorname{Ker} \phi$. Then $H_{1}(\widetilde{X}, \mathbb{C})$ has a structure of $\Lambda$-module where $t$ acts as the canonical covering transformation. Thus by the structure theorem of modules over a principal ideal domain, we have an identification:

$$
H_{1}(\tilde{X}, \mathbb{C}) \cong \Lambda / \lambda_{1} \oplus \cdots \oplus \Lambda / \lambda_{n}
$$

as $\Lambda$-modules. We normalize the denominators so that $\lambda_{i}$ is a polynomial in $t$ with $\lambda_{i}(0) \neq 0$ for each $i=1, \ldots, n$. The Alexander polynomial associated to $\phi$ is defined (see [11]) by the product $\Delta_{\phi}(t):=\prod_{i=1}^{n} \lambda_{i}(t)$.

3.2. Alexander polynomials of plane curves. In our situation, we consider a plane curve $C=C_{1} \cup \cdots \cup C_{r}$ where $C_{1}, \ldots, C_{r}$ are irreducible components of degree $d_{1}, \ldots, d_{r}$ respectively. Take a line $L$ as the line at infinity and let $\phi_{\theta}$ be the composition

$$
\phi_{\theta}: \pi_{1}\left(\mathbb{C}_{L}^{2}-C\right) \stackrel{\xi}{\longrightarrow} H_{1}\left(\mathbb{C}_{L}^{2}-C, \mathbb{Z}\right) \cong \mathbb{Z}^{r} \stackrel{\theta}{\rightarrow} \mathbb{Z}
$$

where $\theta$ is a surjective homomorphism. Recall that $\theta$ is determined by giving an integer $n_{i}$ to each component $C_{i}$ such that $\operatorname{gcd}\left(n_{1}, \ldots, n_{r}\right)=1$. We call $n_{i}$ the weight for $C_{i}$. The Alexander polynomial of $C$ with respect to $(L, \theta)$ is defined by $\Delta_{\phi_{\theta}}(t)$ and we denote it as $\Delta_{C}(t ; L, \theta)$.

(1) (Generic case) Assume that $L$ to be generic and $\theta=\theta_{\text {sum }}$ where $\theta_{\text {sum }}$ is defined by the canonical summation $\theta_{\text {sum }}\left(a_{1}, \ldots, a_{r}\right)=\sum_{i=1}^{r} a_{i}$ (weight 1 for each component.) In this case, we simply write as $\Delta_{C}(t)$ and call it the generic Alexander polynomial of $C$, as it does not depend on the choice of a generic $L$.

(2) If $\theta$ is the canonical summation $\theta_{\text {sum }}$ but $L$ is not generic, we denote it as $\Delta_{C}(t ; L)$, omitting $\theta$. In particular, when $L$ is the tangent line of a smooth point $P \in C$, we call $\Delta_{C}(t ; L)$ the tangential Alexander polynomial at $P$ and we also use the notation $\Delta_{C}(t ; P)$.

(3) If $L$ is generic but $\theta$ is not $\theta_{\text {sum }}$, we called $\Delta_{C}(t ; L, \theta)$ the $\theta$-Alexander polynomial and we denote it as $\Delta_{C}(t ; \theta)$. In 24 we denoted it by $\Delta_{C, \theta}(t)$, but for the consistency of the notation with (2), we use the notation $\Delta_{C}(t ; \theta)$.

Recall that $(t-1)^{r-1} \mid \Delta_{C}(t)([24])$. Thus this is also the case for $\Delta_{C}(t ; L)$ with any line $L$, as $\Delta_{C}(t) \mid \Delta_{C}(t ; L)$. We say that $\Delta_{C}(t: L)$ is trivial if $\Delta_{C}(t ; L)=(t-1)^{r-1}$.

3.3. Fox calculus. Suppose that $G$ is a group and $\phi: G \rightarrow \mathbb{Z}$ is a given surjective homomorphism. Assume that $G$ has a finite presentation as

$$
G \cong\left\langle x_{1}, \ldots, x_{n} \mid R_{1}, \ldots, R_{m}\right\rangle
$$

This corresponds to a surjective homomorphism $\psi: F(n) \rightarrow G$ so that $\operatorname{Ker} \psi$ is normally generated by the words $R_{1}, \ldots, R_{m}$ where $F(n)$ is a free group of rank $n$, generated by 
$x_{1}, \ldots, x_{n}$. Consider the group ring $\mathbb{C}(F(n))$ of $F(n)$ with $\mathbb{C}$-coefficients. The Fox differentials $\frac{\partial}{\partial x_{j}}: \mathbb{C}(F(n)) \rightarrow \mathbb{C}(F(n))$ for $j=1, \ldots, n$, are additive homomorphisms which are characterized by the following properties.
(1) $\frac{\partial}{\partial x_{j}} x_{i}=\delta_{i, j}$
(2) (Leibniz rule) $\frac{\partial}{\partial x_{j}}(u v)=\frac{\partial u}{\partial x_{j}}+u \frac{\partial v}{\partial x_{j}}, u, v \in \mathbb{C}(F(n))$

The composition $\phi \circ \psi: F(n) \rightarrow \mathbb{Z}$ gives a ring homomorphism $\gamma: \mathbb{C}(F(n)) \rightarrow \mathbb{C}\left[t, t^{-1}\right]$. The Alexander matrix $A$ is an $m \times n$ matrix with coefficients in $\mathbb{C}\left[t, t^{-1}\right]$ and its $(i, j)$-component is given by $\gamma\left(\frac{\partial R_{i}}{\partial x_{j}}\right)$. Then the Alexander polynomial $\Delta_{\phi}(t)$ is defined by the greatest common divisor of $(n-1) \times(n-1)$-minors of $A$. In the case of $G=\pi_{1}(X)$ for some connected topological space $X$, this definition coincides with the previous one (Fox [6] ).

3.3.1. Examples. We gives several examples.

1. Consider the trivial case: $G=\mathbb{Z}^{r}$ and $\phi=\theta_{\text {sum }}$, the canonical one. Then

1-1. $G \cong \mathbb{Z} \cong\left\langle x_{1}\right\rangle$. Then $\Delta(t)=1$.

1-2. If $G=\mathbb{Z}^{r} \cong\left\langle x_{1}, \ldots, x_{r} \mid R_{i, j}=x_{i} x_{j} x_{i}{ }^{-1} x_{j}{ }^{-1}, 1 \leq i<j \leq r\right\rangle$, we have $\Delta(t)=$ $(t-1)^{r-1}$. This follows from the Fox derivation:

$$
\frac{\partial}{\partial x_{i}}\left(x_{i} x_{j} x_{i}^{-1} x_{j}^{-1}\right)=1-x_{i} x_{j} x_{i}^{-1}, \quad \frac{\partial}{\partial x_{j}}\left(x_{i} x_{j} x_{i}^{-1} x_{j}{ }^{-1}\right)=x_{i}-x_{i} x_{j} x_{i}^{-1} x_{j}^{-1} .
$$

2. Let $C=\left\{Y^{2} Z-X^{3}=0\right\}$ and $L_{g e n}=\{Z=Y\}, L=\{Z=0\}$. Note that $(0,1,0)$ is a flex point of $C$ and $L$ is the flex tangent. Then

$$
\begin{gathered}
\pi_{1}\left(\mathbb{C}_{L_{\text {gen }}}^{2}-C\right) \cong \mathbb{Z}, \quad \Delta_{C}\left(t ; L_{\text {gen }}\right)=1 \\
\pi_{1}\left(\mathbb{C}_{L}-C\right)=\left\langle x_{1}, x_{2} \mid x_{1} x_{2} x_{1}=x_{2} x_{1} x_{2}\right\rangle \cong B_{3}, \quad \Delta_{C}(t ; L)=t^{2}-t+1
\end{gathered}
$$

3. Let us consider the curve $C=\left\{Y^{2} Z^{3}-X^{5}=0\right\} \subset \mathbb{C}^{2}$ and $L=\{Z=0\}, M=\{Y=0\}$. Then $\pi_{1}\left(\mathbb{P}^{2}-C\right) \cong \mathbb{Z} / 5 \mathbb{Z}$ and $\pi_{1}\left(\mathbb{C}_{L}^{2}-C\right) \cong G(2,5)$ and $\pi_{1}\left(\mathbb{C}_{M}^{2}-C\right) \cong G(3,5)$. In this case, we get

$$
\Delta_{C}(t)=1, \quad \Delta_{C}(t ; L)=\frac{\left(t^{10}-1\right)(t-1)}{\left(t^{5}-1\right)\left(t^{2}-1\right)}, \quad \Delta_{C}(t ; M)=\frac{\left(t^{15}-1\right)(t-1)}{\left(t^{5}-1\right)\left(t^{3}-1\right)}
$$

3.4. Weakness of the generic Alexander polynomial $\Delta_{C}(t)$. The following Lemma describes the relation between the Alexander polynomial and local singularities.

Lemma 6. (Libgober [11]) Let $P_{1}, \ldots, P_{k}$ be the singular points of $C$ (including those at infinity) and let $\Delta_{i}(t)$ be the characteristic polynomial of the Milnor fibration of the germ $\left(C, P_{i}\right)$. Then the generic Alexander polynomial satisfies the divisibility: $\Delta_{C}(t ; L) \mid \prod_{i=1}^{k} \Delta_{i}(t)$.

Lemma 7. (Libgober [1]) Let $d$ be the degree of $C$. Then the Alexander polynomial $\Delta_{C}\left(t ; L_{\infty}\right)$ divides the Alexander polynomial at infinity $\Delta_{\infty}(t)$. If $L_{\infty}$ is generic, $\Delta_{\infty}(t)$ is given by $\left(t^{d}-1\right)^{d-2}(t-1)$. In particular, the roots of the generic Alexander polynomial are $d$-th roots of unity.

Corollary 8. (11], See also [36]) Assume that $C$ is an irreducible curve of degree $d$ and assume that the singularities are either nodes (i.e., $A_{1}$ ) or ordinary cusp singularities (i.e., $\left.A_{2}\right)$. If $d$ is not divisible by 6 , the generic Alexander polynomial $\Delta_{C}(t)$ is trivial. 
This implies that there does not exist any non-trivial generic Alexander polynomials of degree $n$ with $n \not \equiv 0 \bmod 6$, for example, this is the case for cubic, quartic and quintic curves, whose singularities are copies of $A_{1}$ or $A_{2}$. However even though there does exist interesting geometry on these curves. We will show by examples that certain tangential Alexander polynomial gives non-trivial Alexander invariants and we will give an explanation from viewpoint of non-reduced degeneration in $\S 5$.

Another weakness of generic Alexander polynomials is for reducible curves. Let $C_{1}$ and $C_{2}$ be curves which intersect transversely each other. We take a line $L$ so that $L$ does not contain any points of $C_{1} \cap C_{2}$. Note that $L$ need not be generic for $C_{1} \cup C_{2}$. Theorem 4 says that

$$
\pi_{1}\left(\mathbb{C}_{L}^{2}-C_{1} \cup C_{2}\right) \cong \pi_{1}\left(\mathbb{C}_{L}^{2}-C_{1}\right) \times \pi_{1}\left(\mathbb{C}_{L}^{2}-C_{2}\right)
$$

However the Alexander polynomial $\Delta_{C_{1} \cup C_{2}}(t ; L)$ loses these informations. In fact, we have

Theorem 9. (24]) Assume that $C_{1}$ and $C_{2}$ intersect transversely and let $C=C_{1} \cup C_{2}$. Let $L$ be a line such that $L \cap C_{1} \cap C_{2}=\emptyset$. Then $\Delta_{C}(t ; L)=(t-1)^{r-1}$ where $r$ is the number of irreducible components.

For further information about Alexander polynomials, we refer to [7, 11, 13, 14, 31]

\section{Dual stratification and tangential fundamental groups.}

4.1. Dual stratification of curves. Let $\Sigma$ be a finite set of topological equivalent class of curve singularities and let $\mathcal{M}(\Sigma, d)$ be the configuration space of plane curves of degree $d$ with a fixed singularity configuration $\Sigma$. Take two curves $C, C^{\prime} \in \mathcal{M}(\Sigma, d)$ in the same connected component and two smooth points $P \in C$ and $Q \in C^{\prime}$. We consider their tangent lines $L=T_{P} C, L^{\prime}=T_{Q} C$. Though the topology of $\left(\mathbb{P}^{2}, C\right)$ and $\left(\mathbb{P}^{2}, C^{\prime}\right)$ are topologically equivalent, this may not the case for $\left(\mathbb{P}^{2}, C \cup L\right)$ and $\left(\mathbb{P}^{2}, C^{\prime} \cup L^{\prime}\right)$. To analyze this, we introduce the dual stratification $\mathcal{S}(C)$ for $C \in \mathcal{S}(\mathcal{M}(\Sigma, d))$ and $\mathcal{S}(\mathcal{M}(\Sigma, d))$ of $\mathcal{M}(\Sigma, d)$ as follows.

Let $\check{\mathbb{P}}^{2}$ be the dual projective space. Recall that a point $\alpha=\left(\alpha_{1}: \alpha_{2}: \alpha_{3}\right) \in \check{\mathbb{P}}^{2}$ (resp. a point $\left.P=\left(p_{1}: p_{2}: p_{3}\right) \in \mathbb{P}^{2}\right)$ can be considered as a line $L_{\alpha}=\left\{\alpha_{1} X+\alpha_{2} Y+\alpha_{3} Z=0\right\}$ in $\mathbb{P}^{2}$ (resp. a line $L_{P}=\left\{p_{1} U+p_{2} V+p_{3} W=0\right\}$ in $\check{\mathbb{P}}^{2}$ ). First take $C \in \mathcal{M}(\Sigma, d)$. Let $\Sigma(C)=\left\{P_{1}, \ldots, P_{k}\right\}$ be the singular points of $C$. Let $\mathcal{P}(d)$ be the set of partition of the integer $d$. We consider the mappings $\psi: \check{C} \rightarrow \mathcal{P}(d)$ and $\check{\psi}: C \rightarrow \mathcal{P}(\check{d})$ defined as follows. Let $\alpha \in \check{C}$ (resp. $P \in C$ ) and let $L_{\alpha} \cap C=\left\{R_{1}, \ldots, R_{\nu}\right\}$ (resp. $L_{P} \cap \check{C}=\left\{S_{1}, \ldots, S_{\mu}\right\}$ ). We define $\psi(\alpha)=\left\{I\left(C, L_{\alpha} ; R_{i}\right), i=1, \ldots, \nu\right\}$ where $I\left(C, L_{\alpha} ; R_{i}\right)$ is the local intersection multiplicity. Respectively we define $\check{\psi}(P)=\left\{I\left(\check{C}, L_{P} ; S_{j}\right) ; j=1, \ldots, \mu\right\}$. Note that for a generic line $\alpha \in \check{C}, L_{\alpha}$ is a simple tangent line and therefore $\psi(\alpha)=\{2,1, \ldots, 1\}$. For a generic flex point $P$, the tangent line $L=T_{P} C$ gives the partition $\psi(L)=\{3,1, \ldots, 1\}$. A line $\alpha \in \check{C}$ is called an multi-tangent line if $\psi(\alpha)$ has at least two members $\geq 2$. A simple bi-tangent line is a typical such line which is simply tangent at two smooth points. A smooth point $P \in C$ is called tangentially generic if it is smooth and the tangent line $T_{P} C$ gives the partition $\{2,1, \ldots, 1\}$. Recall that the Gauss map associated with $C$, denoted as $G_{C}: C \rightarrow \check{C}$, is defined by $G_{C}(P)=T_{P} C$. Let $\Sigma^{n t g}(C)=\left\{P_{k+1}, \ldots, P_{k+t}\right\}$ be smooth points which are not 
tangentially generic and put $\widetilde{\Sigma}(C)=\Sigma(C) \cup \Sigma^{n t g}(C)=\left\{P_{1}, \ldots, P_{k+t}\right\}$. The dual stratification $\mathcal{S}(C)$ of $C$ is by definition, $\mathcal{S}:=\{C-\widetilde{\Sigma}(C), \widetilde{\Sigma}(C)\}$. Thus if $C$ is irreducible, $\mathcal{S}(C)$ has one open dense stratum made of tangentially generic points and $k+t$ starata made of isolated points.

4.2. Dual stratification of the configuration space $\mathcal{M}(\Sigma, d)$. Now we consider the dual stratification of $\mathcal{M}(\Sigma, d)$. To distinguish a point in $\mathcal{M}(\Sigma, d)$ and the corresponding curve, we denote points in $\mathcal{M}(\Sigma, d)$ by $\alpha \in \mathcal{M}(\Sigma, d)$ and the corresponding curve by $C_{\alpha}$. The configuration of the singularities of the dual curve $\Sigma\left(\check{C_{\alpha}}\right)$ is not unique for $\alpha \in \mathcal{M}(\Sigma, d)$ but it has only finite possible types, say $\Sigma_{1}^{*}, \ldots, \Sigma_{\ell}^{*}$ when we fix the configuration space $\mathcal{M}(\Sigma, d)$. We consider the partition of the configuration space by the following sets:

$$
\left\{\alpha \in \mathcal{M}(\Sigma, d) ;\left(\Sigma\left(\check{C}_{\alpha}\right), \mathcal{S}\left(C_{\alpha}\right), \mathcal{S}\left(\check{C}_{\alpha}\right), \psi_{\alpha}, \check{\psi}_{\alpha}\right) \text { are constant }\right\}
$$

The dual stratification $\mathcal{S}(\mathcal{M}(\Sigma, d))$ is defined by the strata which are the connected components of these partitions. Thus for a stratum $M \in \mathcal{S}(\mathcal{M}(\Sigma, d))$, each $C_{\alpha}$ and $\check{C}_{\alpha}, \alpha \in M$ have constant dual stratifications.

For a stratum $M \in \mathcal{S}(\mathcal{M}(\Sigma, d))$, we can associate a family of plane curves $C_{\alpha}, \alpha \in M$ such that the dual family of curves $\check{C}_{\alpha}, \alpha \in M$ is a family in $\mathcal{M}\left(\Sigma_{j}^{*}, \breve{d}\right)$ for some $j$. Observe that any $\alpha \in M$, the dual stratification $\mathcal{S}\left(C_{\alpha}\right)$ and $\mathcal{S}\left(\check{C}_{\alpha}\right)$ are constant for $\alpha$ by definition. Thus for two $\alpha, \beta \in M, C_{\alpha}, C_{\beta}$ are homeomorphic as a stratified sets. More precisely we have

Proposition 10. Take $\alpha_{0} \in M$ and take a point $L_{\alpha_{0}} \in \check{C}_{\alpha_{0}}$. This induces a continuous family of lines $L_{\alpha} \in \check{C}_{\alpha}$ such that $\psi_{\alpha}\left(L_{\alpha}\right)$ is constant. Then the topology of the affine pair $\left(\mathbb{C}_{L_{\alpha}}^{2}, C\right)$ does not depend on $\alpha \in M$. In particular the fundamental group $\pi_{1}\left(\mathbb{C}_{L_{\alpha}}^{2}-C\right)$ does not depend on $\alpha \in M$.

Proof. Recall that the local topology of $C_{\alpha} \cup L_{\alpha}$ at an intersection point $P$ is determined by the local Milnor number $\mu\left(C_{\alpha} \cup L_{\alpha}, P\right)$ and this is determined by $\mu\left(C_{\alpha}, P\right)$ and the local intersection multiplicity $I\left(C_{\alpha}, L_{\alpha} ; P\right)$. The definition of the dual stratification of $\mathcal{S}(\mathcal{M}(\Sigma, d))$ guarantees the $\mu$-constancy of the family of plane curves $C_{\alpha} \cup L_{\alpha}, \alpha \in M$ of degree $d+1$.

4.3. Tangential fundamental group and Tangential Alexander polynomial. For a line $L \in \check{C}$, we call $\pi_{1}\left(\mathbb{C}_{L}^{2}-C\right)$ the tangential fundamental group and $\Delta_{C}(t ; L)$ the tangential Alexander polynomial. If $L=T_{P} C$ for some simple point $P \in C$, we also use the notation $\Delta_{C}(t ; P)$ for $\Delta_{C}\left(t ; T_{P} C\right)$. We also define $k$-fold Alexander polynomial $\Delta_{C}\left(t ; P_{1}, \ldots, P_{k}\right)$ by $\Delta_{C \cup L_{1} \cup \ldots \cup L_{k-1}}\left(t ; P_{k}\right)$ with $L_{j}=T_{P_{j}} C$. It is easy to observe that $\pi_{1}\left(\mathbb{C}_{L}^{2}-C\right)$ and $\Delta_{C}(t ; P)$ are constant on the open (dense if $C$ is irreducible) strata of the dual stratification $\mathcal{S}(C)$. However in general it may give a different polynomial for singular lines $L \in \check{C}$ (they are the images of isolated strata of $\mathcal{S}(C)$ by the Gauss map). We will see some examples later. Thus the tangential Alexander polynomials altogether contain more geometrical informations than the generic Alexander polynomials. The main purpose of this paper is to investigate the property of the tangential Alexander polynomials. Note that if $C$ is irreducible, there is only one choice of $\theta$ (up to \pm ) but there are many choices for $L$, even for irreducible $C$. 
4.4. Alexander spectrum. We also consider the set of tangential Alexander polynomials

$$
\text { t-AS }(C):=\left\{\Delta_{C}(t ; P) ; P \in C\right\}
$$

and we call t-AS $(C)$ the tangential Alexander spectrum of $C$. There exist at most finite polynomials in the spectrum. In fact, it is bounded by the number of strata of $\mathcal{S}(C)$.

We can also define the $k$-fold tangential Alexander spectrum of $C$ by

$$
\text { t-AS }{ }^{(k)}(C):=\left\{\Delta_{C}\left(t ; P_{1}, \ldots, P_{k}\right) ; P_{j} \in C\right\}
$$

It often happens that even when the Alexander spectrum t-AS $(C)$ is trivial, 2-fold Alexander spectrum t- $\mathrm{AS}^{(2)}(C)$ (or higher one) is not trivial.

4.5. Example. We consider $\mathcal{M}\left(2 A_{2}+A_{1}, 4\right)$ and $\mathcal{M}\left(E_{6}, 4\right)$. By class formula, the dual curve $\check{C}$ of a generic member $C$ of $\mathcal{M}\left(2 A_{2}+A_{1}, 4\right)$ or $\mathcal{M}\left(E_{6}, 4\right)$ is a quartic with $2 A_{2}+A_{1}$ in both cases. ( $C$ has generically 2 flex points.) In both configuration spaces $\mathcal{M}\left(2 A_{2}+A_{1}, 4\right)$ and $\mathcal{M}\left(E_{6}, 4\right)$, there are strata which correspond to degenerated members, namely curves with one flex of order 2. This implies that the dual curve has an $E_{6}$ singularity. For these configuration spaces, there is a beautiful work by C.T.C.Wall [35. Consider the subsets:

$$
\begin{gathered}
M_{1}:=\left\{C \in \mathcal{M}\left(2 A_{2}+A_{1}, 4\right) ; \Sigma(\check{C})=\left\{2 A_{2}+A_{1}\right\}\right\}, \\
M_{2}:=\left\{C \in \mathcal{M}\left(2 A_{2}+A_{1}, 4\right) ; \Sigma(\check{C})=\left\{E_{6}\right\}\right\} \\
N_{1}:=\left\{C \in \mathcal{M}\left(E_{6}, 4\right) ; \Sigma(\check{C})=\left\{2 A_{2}+A_{1}\right\}\right\}, \\
N_{2}:=\left\{C \in \mathcal{M}\left(E_{6}, 4\right) ; \Sigma(\check{C})=\left\{E_{6}\right\}\right\}
\end{gathered}
$$

We can easily see that $\left\{M_{1}, M_{2}\right\},\left\{N_{1}, N_{2}\right\}$ are respective dual stratifications of the configuration spaces $\mathcal{M}\left(2 A_{2}+A_{1}, 4\right)$ and $\mathcal{M}\left(E_{6}, 4\right)$. We observe that under the Gauss map,

$-M_{1}$ and $N_{2}$ are self-dual and

$-M_{2}$ and $N_{1}$ are dual each other.

We observe also that $M_{2} \subset \partial M_{1}$ and $N_{2} \subset \partial N_{1}$.

(1-1) We consider quartic $C_{1} \in M_{1}$ with $\Sigma\left(C_{1}\right)=2 A_{2}+A_{1}$ with two flexes. By the class formula, such a curve has a bi-tangent line. As an example, we take:

$$
C_{1}: \frac{17}{4} y^{4}+8 y^{3}+1 / 4+7 / 2 y^{2}-7 / 2 y^{2} x^{2}+1 / 4 x^{4}-1 / 2 x^{2}=0
$$

$C_{1}$ has two cusps at $P_{1}=(1,0), P_{2}=(-1,0)$ and one $A_{1}$ at $(0,-1)$. Two flexes are at $Q_{1}=(10 \sqrt{10}, 9), Q_{2}=(-10 \sqrt{10}, 9)$. We have a bi-tangent line $y=1$ which are tangent at $B_{1}=(2 \sqrt{2}, 1), Q_{2}=(-2 \sqrt{2}, 1)$. For the dual stratification $\mathcal{S}\left(C_{1}\right)$, we have to take two more points $S_{1}=(25 / 7,8 / 7), S_{2}=(-25 / 7,8 / 7)$ whose tangent lines pass through $P_{2}$ and $P_{1}$ respectively. Using Zariski-van Kampen pencil method, we can compute $\pi_{1}\left(\mathbb{C}_{L}^{2}-C_{1}\right)$ as

$$
\pi_{1}\left(\mathbb{C}_{L}^{2}-C_{1}\right)=\left\langle\xi_{1}, \xi_{2}, \xi_{3} \mid \xi_{1} \xi_{2} \xi_{1}=\xi_{2} \xi_{1} \xi_{2}, \xi_{2} \xi_{3} \xi_{2}=\xi_{3} \xi_{2} \xi_{3}, \xi_{1} \xi_{3}=\xi_{3} \xi_{1}, \xi_{1} \xi_{2} \xi_{3}=\xi_{3} \xi_{2} \xi_{1}\right\rangle
$$

where $L=\{y=1\}$. This gives $\Delta_{C_{1}}(t ; L)=t^{2}-t+1$ by Fox calculus. Other tangent lines give the trivial Alexander polynomial. We leave the proof of this assertion as an exercise. Thus t-AS $\left(C_{1}\right)=\left\{1, t^{2}-t+1\right\}$. 
(1-2) We consider the following quartic $C_{2} \in M_{2}$ with $\Sigma\left(C_{2}\right)=2 A_{2}+A_{1}$ with a degenerated flex of order 2 at infinity $L=\{Z=0\}$ :

$$
\begin{gathered}
C_{2}: \quad 1-12 y+36 y^{2}-32 y^{3}-2 x^{2}+12 x^{2} y+x^{4}=0 \\
\pi_{1}\left(\mathbb{C}_{L}^{2}-C_{2}\right)=\left\langle\xi_{1}, \xi_{2}, \xi_{3} \mid \xi_{1} \xi_{3} \xi_{1}=\xi_{3} \xi_{1} \xi_{3}, \xi_{3} \xi_{2} \xi_{3}=\xi_{2} \xi_{3} \xi_{2}, \xi_{2} \xi_{1}=\xi_{1} \xi_{2}\right\rangle
\end{gathered}
$$

We can also see that $\Delta_{C_{2}}(t ; L)=t^{2}-t+1$. Note that two $A_{2}$ singularities are at $( \pm 1,0)$ and one $A_{1}$ is at $(0,1 / 2)$. In the dual stratification $\mathcal{S}\left(C_{2}\right)$, there are two more 'singular' points $R_{1}=(9,8)$ and $R_{2}=(-9,8)$ whose tangent line pass through the cusps. However these tangent lines give trivial tangential Alexander polynomials.

(2-1) Consider a quartic $C_{3} \in N_{1}$, defined by $y^{3}+x^{4}-x^{2} y^{2}=0$ which has one $E_{6}$-singularity at $O$. Two flex points are at $( \pm 6 \sqrt{6} / 5,36 / 5)$. The bi-tangent line is given by $y=4$. By an easy computation, we observe that $L=\{y=4\}$ gives $\pi_{1}\left(\mathbb{C}_{L}^{2}-C_{3}\right) \cong B_{3}$ and $\Delta_{C_{3}}(t ; L)=$ $t^{2}-t+1$. Other tangent Alexander spectra are trivial. So $t-\operatorname{AS}\left(C_{3}\right)=\left\{1, t^{2}-t+1\right\}$. The dual stratification has 5 isolated points.

(2-2) Consider the following quartic $C_{4} \in N_{2}, f(x, y)=y^{3}+x^{4}=0$, with $E_{6}$ and one flex of order 2 at $P=(0,1,0)$. Take the flex line $Z=0$ as $L$. Then

$$
\pi_{1}\left(\mathbb{C}_{L}^{2}-C\right) \cong G(3,4)=\left\langle\xi_{0}, \xi_{1}, \xi_{2} \mid \xi_{0}=\omega \xi_{1} \omega^{-1}, \xi_{1}=\omega \xi_{2} \omega^{-1}\right\rangle
$$

and $\Delta_{C_{4}}(t ; L)=\left(t^{2}-t+1\right)\left(t^{4}-t^{2}+1\right)$ where $\omega=\xi_{2} \xi_{1} \xi_{0}$.

(3) Let $C: f(x, y)=-\frac{1}{2} y^{4}-\frac{1}{2}-3 x^{2} y^{2}+y^{2}+\frac{3}{2} x^{4}-4 x^{3}+3 x^{2}=0$ be a 3 cuspidal quartic. As is well-known [36, 19], the generic affine fundamental group is a finite group of order 12, with presentation

$$
\pi_{1}\left(\mathbb{C}^{2}-C\right)=\left\langle\xi, \zeta \mid \xi \zeta \xi=\zeta \xi \zeta, \xi^{2}=\zeta^{2}\right\rangle
$$

Though the fundamental group is not abelian, the generic Alexander polynomial is trivial. For $L=\{y=0\}$ (this is the tangent cone of a cusp and $L$ corresponds to a flex of $\check{C}$ ),

$$
\pi_{1}\left(\mathbb{C}_{L}^{2}-C\right)=\langle\xi, \zeta \mid \xi \zeta \xi=\zeta \xi \zeta\rangle=B_{3}
$$

By the class formula, the dual curve $\check{C}$ is a cubic curve with a node. $\mathcal{S}(C)$ has 3 singular points from $3 A_{2}$ and two 'singular points' from the bi-tangent line. We can also see that $\pi_{1}\left(\mathbb{C}_{L}^{2}-C\right) \cong B_{3}$ for an arbitrary tangent line $L$ except the bitangent line $L_{b}$. The bi-tangent line is given by $x=2 / 3$ in this example. By an easy computation, we see that

$$
\pi_{1}\left(\mathbb{C}_{L_{b}}^{2}-C\right) \cong\left\langle\xi_{0}, \xi_{1}, \xi_{2}, \zeta \mid \xi_{0} \xi_{1} \xi_{0}=\xi_{1} \xi_{0} \xi_{1}, \xi_{1} \xi_{2} \xi_{1}=\xi_{2} \xi_{1} \xi_{2}, \xi_{2} \zeta \xi_{2}=\zeta \xi_{2} \zeta, \zeta=\xi_{1}^{-1} \xi_{0} \xi_{1}\right\rangle
$$

and we have

Proposition 11. For the bitangent line $L_{b}, \Delta_{C}\left(t ; L_{b}\right)=\left(t^{2}-t+1\right)^{2}$. For any other tangent line $L, \Delta_{C}(t ; L)=t^{2}-t+1$. In particular, this implies that $t-A S(C)=\left\{t^{2}-t+1,\left(t^{2}-t+1\right)^{2}\right\}$. 
4.5.1. Further example. In the above examples of quartics, the geometry of $C \cup T_{P} C$ does not change for flexes of the same order. However this is not the case in general.

Consider a fixed mark point $P \in C$. We call $(C, P)$ a curve with a marked point $P$. Two curves with marked points $(C, P)$ and $\left(C^{\prime}, P^{\prime}\right)$ are called a marked Zariski pair if $\left\{C \cup T_{P} C, C^{\prime} \cup T_{P^{\prime}} C^{\prime}\right\}$ is a Zariski pair. For further information about Zariski pairs, see [1, 2, 3, 24, 25]. In [26], we have shown that for any quintic $B_{5}$ with configuration in the next list, there exist two different flex points $P, P^{\prime}$ such that $\left(B_{5}, P\right)$ and $\left(B_{5}, P^{\prime}\right)$ are marked Zariski pairs.

$$
\left\{\begin{array}{l}
4 A_{2}, 4 A_{2}+A_{1}, A_{5}+2 A_{2}, A_{5}+2 A_{2}+A_{1}, E_{6}+2 A_{2} \\
E_{6}+A_{5}, 2 A_{5}, A_{8}+A_{2}, A_{8}+A_{2}+A_{1}, A_{11}
\end{array}\right.
$$

In fact their generic Alexander polynomials are given as $t^{2}-t+1,1$ respectively. This implies that the among flexes of these quintics, there are two classes of different topological nature: one class which does not contribute the tangential Alexander spectrum and the other which contributes by $\left(t^{2}-t+1\right)$. We give one example. The following quintic $B_{5}: f(x, y)=0$ has $A_{11}$ singularity at the origin and 9 flex points. Among them, the flex at $P=(0,1)$ is different from others (a flex of torus type). In fact, $B_{5} \cup T_{P} B_{5}$ is a sextic of torus type [26]. All other flex points gives trivial tangential Alexander polynomial.

$$
\begin{array}{r}
f(x, y)=-\frac{33}{64} y^{5}+\left(\frac{7}{8} x+\frac{129}{64}\right) y^{4}+\left(-\frac{5}{4} x^{2}-\frac{15}{8} x-5 / 2\right) y^{3}+ \\
\left(\frac{15}{8} x^{3}+\frac{13}{4} x^{2}+x+1\right) y^{2}+\left(-\frac{3}{4} x^{4}-2 x^{3}-2 x^{2}\right) y+x^{5}+x^{4}
\end{array}
$$

Figure 1. Quintic with $A_{11}$

4.6. $\theta$-Alexander polynomials. To cover the weakness of Alexander polynomials for irreducible curves, we have proposed $\theta$-Alexander polynomials in 24. First recall that the radical $\sqrt{q(t)}$ of a polynomial $q(t)=\prod_{i=1}^{\nu}\left(t-\xi_{i}\right)^{\mu_{i}}$ is defined by $\sqrt{q(t)}:=\prod_{i=1}^{\nu}\left(t-\xi_{i}\right)$. Here $\mu_{i} \geq 1, \forall i$. The following theorem shows the importance of $\theta$-Alexander polynomial. 
Theorem 12. (24) Assume that $C, C^{\prime}$ be reduced curves and we assume further $C^{\prime}$ is irreducible. For a given integer $n$, suppose that the surjective homomorphism $\phi_{n}: \pi_{1}\left(\mathbb{C}_{L}^{2}-C \cup C^{\prime}\right)$ $\rightarrow \mathbb{Z}$ which has weight 1 on each component of $C$ and weight $n$ on $C^{\prime}$. Then

(1) $\Delta_{C \cup C^{\prime}}\left(t ; L, \phi_{n}\right)$ is divisible by $\operatorname{gcd}\left(\Delta_{C}(t ; L) \times(t-1),\left(t^{n}-1\right)\right)$ for $n \neq 0$.

Suppose further that $C \pitchfork C^{\prime}$ and $\pi_{1}\left(\mathbb{C}_{L}^{2}-C^{\prime}\right) \cong \mathbb{Z}$. Then

(2) $\Delta_{C}(t ; L) \times(t-1)$ is divisible by $\Delta_{C \cup C^{\prime}}\left(t ; L, \phi_{n}\right)$.

(3) $\sqrt{\Delta_{C \cup C^{\prime}}\left(t ; L, \phi_{n}\right)}=\operatorname{gcd}\left(\Delta_{C}(t ; L) \times(t-1),\left(t^{n}-1\right)\right)$.

(4) In particular, if $\operatorname{gcd}\left(\Delta_{C}(t ; L) \times(t-1),\left(t^{n}-1\right)\right)=\Delta_{C}(t ; L)$, we have

$$
\Delta_{C \cup C^{\prime}}\left(t ; L, \phi_{n}\right)=\Delta_{C}(t ; L) \times(t-1) .
$$

Proof. The proof goes exactly as in [24]. Consider the canonical surjective homomorphism: $h: \pi_{1}\left(\mathbb{C}_{L}^{2}-C \cup C^{\prime}\right) \rightarrow \pi_{1}\left(\mathbb{C}_{L}^{2}-C\right) \times \mathbb{Z}$. Consider the presentation.

$$
\pi_{1}\left(\mathbb{C}_{L}^{2}-C\right)=\left\langle g_{1}, \ldots, g_{s} \mid R_{1}, \ldots, R_{k}\right\rangle, s \leq \operatorname{degree} C
$$

The homomorphism $\phi_{n}$ factors as $\phi_{n}=\psi \circ h$ where $\psi$ is the surjective homomorphism $\psi: \pi_{1}\left(\mathbb{C}_{L}^{2}-C\right) \times H_{1}\left(\mathbb{C}_{L}^{2}-C^{\prime}\right) \cong \pi_{1}\left(\mathbb{C}_{L}^{2}-C\right) \times \mathbb{Z} \rightarrow \mathbb{Z}$ where the weight of the second factor is $n$. Note that $\Delta_{C \cup C^{\prime}}\left(t ; L, \phi_{n}\right)=\Delta_{\phi_{n}}(t)$ in the notation of $\S 3.1$. By the above factorization, we have the divisibility: $\Delta_{\psi} \mid \Delta_{\phi_{n}}$. Now the calculation of $\Delta_{\psi}$ is done in the exact same manner as in 24]. We use the presentation:

$$
\pi_{1}\left(\mathbb{C}_{L}^{2}-C\right) \times \mathbb{Z}=\left\langle g_{1}, \ldots, g_{s}, \xi \mid R_{1}, \ldots, R_{k}, T_{i}, 1 \leq i \leq s\right\rangle
$$

where $T_{i}=g_{i} \xi g_{i}^{-1} \xi^{-1}$. The key point of the calculation is the following:

$$
\frac{\partial T_{i}}{\partial g_{i}}=1-g_{i} \xi g_{i}^{-1} \mapsto 1-t^{n}, \quad \frac{\partial T_{i}}{\partial \xi}=g_{i}-g_{i} \xi g_{i}^{-1} \xi^{-1} \mapsto t-1
$$

Let $M$ be the Alexander matrix of $\theta_{\text {sum }}: \pi_{1}\left(\mathbb{C}_{L}^{2}-C\right) \rightarrow \mathbb{Z}$ and let $M^{\prime}$ be the Alexander matrix of $\psi: \pi_{1}\left(\mathbb{C}_{L}^{2}-C\right) \times \mathbb{Z} \rightarrow \mathbb{Z}$. Then $M^{\prime}$ is written as

$$
M^{\prime}=\left(\begin{array}{cc}
M & \overrightarrow{0} \\
N_{1} & N_{2}
\end{array}\right)
$$

where $\overrightarrow{0}$ is a zero vector and $N_{1}$ is a $s \times s$-matrix which is explicitly given as $\left(1-t^{n}\right) E_{s}$ where $E_{s}$ is the identity matrix of rank $s$. The vector $N_{2}$ takes the form ${ }^{t}(t-1, \ldots, t-1)$ where $t-1$ is repeated $s$ times. For any $(s-1) \times(s-1)$-minor $B$ of $M$, let $\tilde{B}$ be the $s \times s$-minor adding $(k+1)$-th row and the last column. Then $\operatorname{det} \tilde{B}=\operatorname{det} B \times(t-1)$. Thus we have $\Delta_{C}(t ; L) \times(t-1)$ as the common divisor of such det $\tilde{B}$ 's. Also we get $\left(t^{n}-1\right)^{s}$ by taking a minor from $N_{1}$. We observe also that any determinant of a $s \times s$-minor which contains at least two rows of $\left(N_{1}, N_{2}\right)$ is divisible by $\left(t^{n}-1\right)$. Thus we observe two divisibilities:

$$
\Delta_{\psi}(t)\left|\operatorname{gcd}\left(\Delta_{C}(t ; L) \times(t-1),\left(t^{n}-1\right)^{s}\right), \quad \operatorname{gcd}\left(\Delta_{C}(t ; L) \times(t-1),\left(t^{n}-1\right)\right)\right| \Delta_{\psi}(t)
$$

Note that $\Delta_{\psi}(t) \mid \Delta_{C \cup C^{\prime}}\left(t ; \phi_{n}, L\right)$, by the usual degeneration argument 24]. Thus the first assertion is immediate from the last divisibility. Suppose further that $C \pitchfork C^{\prime}$ and $\pi_{1}\left(\mathbb{C}_{L}^{2}-\right.$ $\left.C^{\prime}\right) \cong \mathbb{Z}$. Then $h$ is an isomorphism and therefore $\Delta_{C \cup C^{\prime}}\left(t ; \phi_{n}, L\right)=\Delta_{\psi}(t)$. Thus the assertions (2), (3) follow immediately. The assertion (4) is a result of (1) and (2). 
4.7. Relations between the tangential Alexander polynomials and $\theta$-Alexander polynomials. Let $C$ be a plane curve of degree $d$ and let $P \in C$ and let $L=T_{P} C$. We consider the tangential Alexander polynomial $\Delta_{C}(t ; L)$. Let

$$
\pi_{1}\left(\mathbb{C}_{L}^{2}-C\right) \cong\left\langle g_{1}, \ldots, g_{d} \mid R_{1}, \ldots, R_{\ell}\right\rangle
$$

be a presentation of $\pi_{1}\left(\mathbb{C}_{L}^{2}-C\right)$ by generators and relations. Take a generic line $L_{\infty}$ for $C \cup L$ and put $\mathbb{C}^{2}=\mathbb{P}^{2}-L_{\infty}$ as usual. Then by Theorem 4 , we have

$$
\pi_{1}\left(\mathbb{C}^{2}-C \cup L\right)=\pi_{1}\left(\mathbb{C}_{L}^{2}-C \cup L_{\infty}\right) \cong \pi_{1}\left(\mathbb{C}_{L}^{2}-C\right) \times \pi_{1}\left(\mathbb{C}_{L}^{2}-L_{\infty}\right) \cong \pi_{1}\left(\mathbb{C}_{L}^{2}-C\right) \times \mathbb{Z}
$$

and it has a presentation:

$$
\begin{aligned}
\pi_{1}\left(\mathbb{C}^{2}-C \cup L\right) & =\left\langle g_{1}, \ldots, g_{d}, h, h_{\infty} \mid R_{1}, \ldots, R_{\ell}, T_{1}, \ldots, T_{d}, S\right\rangle \\
& =\left\langle g_{1}, \ldots, g_{d}, h_{\infty} \mid R_{1}, \ldots, R_{\ell}, T_{1}, \ldots, T_{d}\right\rangle
\end{aligned}
$$

where $T_{j}=h_{\infty} g_{j} h_{\infty}^{-1} g_{j}^{-1}$ and $S=h h_{\infty} g_{d} \ldots g_{1}$. Now the tangential Alexander polynomial $\Delta_{C}(t ; L)$ is associated to the surjective homomorphism

$$
\theta_{\text {sum }}: \pi_{1}\left(\mathbb{C}_{L}^{2}-C\right) \rightarrow \mathbb{Z}=\langle t\rangle, g_{i} \mapsto t
$$

Let $\theta_{n}$ be the surjective homomorphism with weight $n$ on $L$

$$
\theta_{n}: \pi_{1}\left(\mathbb{C}^{2}-C \cup L\right) \longrightarrow \mathbb{Z}=\langle t\rangle, g_{i} \mapsto t, h \mapsto t^{n}
$$

Now taking $g_{1}, \ldots, g_{k}, h_{\infty}$ as generators of $\pi_{1}\left(\mathbb{C}^{2}-C \cup L\right)=\pi_{1}\left(\mathbb{C}_{L}^{2}-C \cup L_{\infty}\right), \theta_{n}$ corresponds to the homomorphism:

$$
\eta_{n+d}: \pi_{1}\left(\mathbb{C}_{L}^{2}-C \cup L_{\infty}\right) \rightarrow \mathbb{Z}, \quad g_{i} \mapsto t, \quad h_{\infty} \mapsto t^{-n-d}
$$

The last property is the result of the observation: $1=\theta_{n}(S)=t^{d+n} \theta_{n}\left(h_{\infty}\right)$.

Notation. Hereafter we mainly consider the weight like $\theta_{n}$ which has weight one except a line component $L$ in consideration. So we introduce the following notation which is easier to be understood:

$$
\Delta_{C \cup L^{n}}(t):=\Delta_{C \cup L}\left(t ; \theta_{n}\right)
$$

The upper index $n$ implies that $L$ has weight $n$. Using this notation, we also write $\Delta_{C \cup L_{\infty}}\left(t ;, \eta_{n+d}, L\right)=\Delta_{C \cup L_{\infty}^{-n-d}}(t ; L)$.

Thus combining the above argument with Theorem 12 we have shown the following.

Theorem 13. For any integer $n, \Delta_{C \cup L^{n}}(t)=\Delta_{C \cup L_{\infty}^{-n-d}}(t ; L)$ and we have the divisibility:

$$
\Delta_{C \cup L_{\infty}^{-n-d}}(t ; L) \mid \Delta_{C}(t ; L) \times(t-1) \quad \text { and } \quad \operatorname{gcd}\left(\Delta_{C}(t ; L) \times(t-1),\left(t^{n+d}-1\right)\right) \mid \Delta_{C \cup L_{\infty}^{-n-d}}(t ; L)
$$

Furthermore if $\operatorname{gcd}\left(\Delta_{C}(t ; L) \times(t-1),\left(t^{n+d}-1\right)\right)=\Delta_{C}(t ; L)$, we have the equality:

$$
\Delta_{C}(t ; L) \times(t-1)=\Delta_{C \cup L_{\infty}^{-n-d}}(t ; L) .
$$


4.7.1. Examples. Let $C$ be a quartic with either $2 A_{2}+A_{1}$ or $E_{6}$ and one flex of order 2. Let $L$ be the flex tangent line. Then we have shown that $\Delta_{C}(t ; L)=t^{2}-t+1$ and $\left(t^{2}-t+1\right)\left(t^{4}-t^{2}+1\right)$ respectively. We can compute generic Alexander polynomials $\Delta_{C \cup L^{2}}(t)$ of $C \cup L$ as follows. Take a generic line $L_{\infty}$.

(1) $C$ is a quartic with $2 A_{2}+A_{1}$ and $L$ is the flex tangent line. As $\Delta_{C}(t ; L)=t^{2}-t+1$, we take weight $n=2$ on $L$ and by Theorem 12, we get

$$
\Delta_{C \cup L_{\infty}^{-6}}(t ; L)=\Delta_{C \cup L^{2}}(t)=\left(t^{2}-t+1\right)(t-1)
$$

Let $C_{t}$ be a family of quartics with $2 A_{2}+A_{1}$ with two flex points for $t \neq 0$ and $C_{0}=C$. Let $L_{1}, L_{2}$ be two tangent lines at the flex points of $C_{t}$. Then $C_{t}+L_{1}+L_{2} \rightarrow C+2 L$. Thus the weight 2 on $L$ is canonical. See $\S 5.7$.

(2) $C$ is a quartic with $E_{6}$ and $L$ is the flex tangent line at a flex of order 2 . As $\Delta_{C}(t ; L)=$ $\left(t^{2}-t+1\right)\left(t^{4}-t^{2}+1\right), \operatorname{gcd}\left(\sqrt{\Delta_{C}(t ; L)},\left(t^{12}-1\right)\right)=\Delta_{C}(t ; L)$. Thus we take $n=8$.

$$
\Delta_{C \cup L^{8}}(t)=\Delta_{C \cup L_{\infty}^{-12}}(t ; L)=(t-1)\left(t^{2}-t+1\right)\left(t^{4}-t^{2}+1\right)
$$

This can be interpreted as $Y^{3} Z+X^{4}=0$ is a line degeneration of (3,4)-torus curve of degree 12 as $\left(Y^{3} Z+X^{4}\right) Z^{8}=\left(Y Z^{3}\right)^{3}+\left(X Z^{2}\right)^{3}$. See $\S 5.4$. Note also that

$$
\Delta_{C \cup L^{2}}(t)=\Delta_{C \cup L_{\infty}^{-6}}(t ; L)=(t-1)\left(t^{2}-t+1\right) .
$$

We can also interpret this equality as a result of a line degeneration of (2,3)-sextics of torus type as $\left(Y^{3} Z+X^{4}\right) Z^{2}=(Y Z)^{3}+\left(X^{2} Z\right)^{2}$.

\section{Degeneration into NOn-Reduced CURVES With a Multiple Line}

In this section, we study an analytic family of curves $C_{t}, t \in \Delta$ such that $C_{0}$ is not reduced but it has a line component with multiplicity.

5.1. Admissible polydisk. Consider a reduced curve $C \subset \mathbb{P}^{2}$ which is defined by a polynomial $f(x, y)=0$ in the affine space $\mathbb{C}_{L}^{2}:=\mathbb{P}^{2}-L$ where $L=\{Z=0\}$. (We do not assume the genericity of the line $L$.) We assume that $f(x, y)$ is a polynomial in $y$ of degree $n$. The base point of the pencil $\left\{L_{\eta}, \eta \in \mathbb{C}\right\}$ where $L_{\eta}:=\{X-\eta Z=0\}$ is given by $B=(0,1,0)$ in the homogeneous coordinates. Note that $n<d$ if and only if $B \in C$. We say the pencil $\{x=\eta, \eta \in \mathbb{C}\}$ is base point admissible (respectively base point non-admissible) if $n=d$ (resp. $n<d)$. Recall that $L_{\eta}$ is a singular line for $C$ if $L_{\eta} \cap C \cap \mathbb{C}_{L}^{2}$ contains some non-transverse intersection point. For the case $n<d$, we also call $L_{\eta}$ singular if the number of the points $L_{\eta} \cap C \cap \mathbb{C}_{L}^{2}$ counted with multiplicity is strictly less than $n$. In this case, we say $L_{\eta}$ a singular line with disappeared points at infinity. Using Zariski-van Kampen pencil method with respect to the pencil lines $x=\eta, \eta \in \mathbb{C}$, we get a presentation

$$
\pi_{1}\left(\mathbb{C}_{L}^{2}-C\right)=\left\langle g_{1}, \ldots, g_{n} \mid R_{1}, \ldots, R_{m}\right\rangle
$$

We consider the polydisk $\Delta_{\alpha, \beta}:=\Delta_{\alpha} \times \Delta_{\beta}$ where $\Delta_{\alpha}:=\{x|| x \mid \leq \alpha\}$ and $\Delta_{\beta}:=\{y|| y \mid \leq \beta\}$ and we consider the following conditions. 
(1) For any $\eta \in \Delta_{\alpha}, L_{\eta} \cap C \subset\{\eta\} \times \Delta_{\beta / 2}$ and $L_{\eta}$ does not have any disappeared points at infinity and

(2) For any $\eta \in \partial \Delta_{\alpha}, L_{\eta}$ is not a singular line.

(3) For any singular line $L_{\eta}, \eta \in \Delta_{\alpha}$.

We say that the polydisk $\Delta_{\alpha, \beta}$ is admissible for $C$ with respect to $L$ if it satisfies (1) and (2). Furthermore, we say that the polydisk $\Delta_{\alpha, \beta}$ is topologically presenting for $C$ with respect to $L$ if it satisfies (1), (2) and (3). Observe that if $\Delta_{\alpha, \beta}$ is topologically presenting for $C$ with respect to $L$, the inclusion $\Delta_{\alpha, \beta}-C \cap \Delta_{\alpha, \beta} \hookrightarrow \mathbb{C}_{L}^{2}-C$ is a homotopy equivalence.

5.2. Two non-reduced degenerations. In this section, we focus the following two types of non-reduced degenerations.

Type 1: Line Degenerations. $\left\{C_{t}, t \in \Delta\right\}$ is an analytic family of irreducible curves of degree $d$ and it degenerate into $C_{0}=D_{0}+j L_{\infty}, j \geq 0$ where $D_{0}$ is an irreducible curve of degree $d-j$ and $L$ is a line. We assume also

$(\sharp)$ there is a point $Q \in L-L \cap D_{0}$ such that $Q \in C_{t}, \forall t \neq 0$ and the multiplicity of $\left(C_{t}, Q\right)$ is $j$.

We call such a degeneration a line degeneration of order $j$. $L$ and $Q$ are called the limit line and the base point of the degeneration respectively. Here $j$ is a non-negative integer. (We are mainly interested in the case $j \geq 2$.) The condition $(\sharp)$ can be weakened as

$\left(\sharp^{\prime}\right)$ there is an analytic family of points $Q_{t} \in L \cap C_{t}$ such that $Q_{0} \in L-L \cap D_{0}$

and the multiplicity of $\left(C_{t}, Q_{t}\right)$ is $j$.

In fact, under $\left(\sharp^{\prime}\right)$, we may assume that $L=\{Z=0\}$ and $Q_{t}=(\alpha(t), 1,0)$. Then taking a linear change of coordinates $(x, y) \mapsto(x, y+\alpha(t) x)$, we can assume that $Q_{t} \equiv(0,1,0)$. We recall that $\mathrm{O}$. Zariski observed that 3 cuspidal quartic is a non-reduced line degeneration of order 2 from a family of sextics of torus type [36].

Type 2: Flex Degenerations. First we have a family of reduced curves $\left\{C_{t}, t \in \Delta\right\}$ of the same degree. $C_{0}$ can be a degeneration if $\mu\left(C_{0}\right)>\mu\left(C_{t}\right), t \neq 0$ or in the same configuration space if $\mu\left(C_{t}\right)=\mu\left(C_{0}\right)$ but in this case, $C_{0}$ is in a different stratum of the dual stratification. On $C_{t}$, we are given flex lines $L_{1}(t), \ldots, L_{k}(t), k \geq 2$ such that the family $L_{i}(t)$ is an analytic family with $L_{i}(0)=L$ for $i=1, \ldots, k$. We associate a non-reduced degeneration $C_{t}+L_{1}(t)+\cdots+L_{k}(t) \rightarrow C_{0}+k L$ and we call this family a flex degeneration.

5.3. Surjectivity Theorem for line degenerations. Assume first that we have an analytic family of curves $\left\{C_{t},|t| \leq 1\right\}$ such that $C_{t}$ is an irreducible curve of degree $d$ and it degenerates into $C_{0}=D_{0}+j L$ where $D_{0}$ is an irreducible curve of degree $d-j$ and $L$ is a line. We assume that $\left\{C_{t}, t \neq 0\right\}$ has $Q=(0,1,0)$ as the base point and the multiplicity of $C_{t}$ at $Q$ is constantly equal to $j$. Let $F(X, Y, Z, t)=0$ be the defining homogeneous polynomial of degree $d$. We assume that $L=\{Z=0\}$. By the assumption, we have $F(X, Y, Z, 0)=Z^{j} G(X, Y, Z)$ where degree $G(X, Y, Z)=d-j$. Put $f(x, y, t):=F(x, y, 1, t)$. This is the affine equation of $C_{t}$. Note that

(1) degree $_{\{x, y\}} f(x, y, t)=d$ for $t \neq 0$

(2) degree $_{\{x, y\}} f(x, y, 0)=d-j$ and degree $_{y} f(x, y, t)=d-j$ for any $t$. 
The second assertion follows from Bézout theorem and the assumption that $C_{t}$ has multiplicity $j$ at $Q$.

Theorem (Surjectivity) 14. Under the above assumption, there is a canonical surjection

$$
\phi: \pi_{1}\left(\mathbb{C}_{L}^{2}-D_{0}\right) \rightarrow \pi_{1}\left(\mathbb{C}_{L}^{2}-C_{\tau}\right), \quad \tau \neq 0, \text { sufficiently small }
$$

Proof. Note that the linear system $L_{\eta}=\{x=\eta z\}, \eta \in \mathbb{C}$ has the base point $Q=(0,1,0)$. Suppose that we have chosen a topologically presenting polydisk $\Delta_{\alpha, \beta}$ for $D_{0}$. Let $f(x, y, \tau)$ be the defining affine polynomial for $C_{\tau}$. As the effect of the non-reducedness disappears when we put $z=1$ in $F(X, Y, Z, t), f(x, y, t)$ is a analytic family. Write $f(x, y, t)$ as

$$
f(x, y, t)=a_{d-j}(x, t) y^{d-j}+\cdots+a_{0}(x, t)
$$

By continuity, we can assume that this polydisk is also admissible for $C_{\tau}$ for some $\delta$ and $|\tau| \leq \delta$. Let $\eta_{1}, \ldots, \eta_{\nu}$ be the parameters corresponding to the singular lines for $D_{0}$. We take a small positive nuber $\varepsilon$ so that the disks $\Delta_{\varepsilon}\left(\eta_{i}\right):=\left\{\eta|| \eta-\eta_{i} \mid \leq \varepsilon\right\}, i=1, \ldots, \nu$ are disjoint each other and $\Delta_{\varepsilon}\left(\eta_{i}\right) \subset \Delta_{\alpha}$. By the assumption, we have $\left|\eta_{j}\right|<\alpha$ for each $j$. We choose a generic pencil line $L_{\eta_{0}}$ and generators $g_{1}, \ldots, g_{d}$ in this pencil so that we have a presentation:

$$
\pi_{1}\left(\mathbb{C}_{L}^{2}-D_{0}\right) \cong\left\langle g_{1}, \ldots, g_{d} \mid R_{1}, \ldots, R_{m}\right\rangle
$$

For sufficiently small $\tau$, the original singular pencil $L_{\eta_{i}}$ for $D_{0}$ may splits into several singular lines for the curves $C_{\tau}, \tau \neq 0$ but they are inside $\Delta_{\varepsilon}\left(\eta_{i}\right)$. Put the corresponding parameters $\eta_{i, 1}, \ldots, \eta_{i, \nu_{i}}$. Note that the monodromy relations around $L_{\eta_{i}}$ for $D_{0}$ is nothing but the product of the monodromy relations around $L_{\eta_{i, s}}$ for $s=1, \ldots, \nu_{i}$ under a suitable ordering. This is immediate from the topological stability of the pencil restricted on the circle $\partial \Delta_{\varepsilon}\left(\eta_{i}\right)$. Note also that $C_{\tau}$ may have a singular line $L_{\eta}$ such that $|\eta| \rightarrow \infty$ when $\tau \rightarrow 0$. Anyway we can get a presentation by adding several more relations $R_{m+1}, \ldots, R_{m+n}$ to (2) (using the same generators $\left.g_{1}, \ldots, g_{d}\right)$ :

$$
\pi_{1}\left(\mathbb{C}_{L}^{2}-C_{\tau}\right)=\left\langle g_{1}, \ldots, g_{d} \mid R_{1}, \ldots, R_{m}, R_{m+1}, \ldots, R_{m+n}\right\rangle
$$

This and (2) implies that there is a canonical surjection $\phi: \pi_{1}\left(\mathbb{C}_{L}^{2}-D_{0}\right) \rightarrow \pi_{1}\left(\mathbb{C}_{L}^{2}-C_{\tau}\right)$.

Remark 15. Though we are mainly concerned in the case $j \geq 2$, the assertion for $j=0$ gives another proof of Theorem 3.

Corollary 16. Under the same assumption, we have the divisibility $\Delta_{C_{\tau}}(t ; L) \mid \Delta_{D_{0}}(t ; L)$.

Taking a generic line $L_{\infty}$ for $C_{\tau}, \tau$ small and $D_{0} \cup L$, we apply Theorem 13 to obtain:

Corollary 17. Under the same assumption,

$$
\sqrt{\Delta_{C_{\tau}}(t)} \mid \Delta_{D_{0} \cup L^{j}}(t)
$$

Proof. First note that $\Delta_{C_{\tau}}(t) \mid \Delta_{C_{\tau}}(t ; L)$ and $\Delta_{C_{\tau}}(t ; L) \mid \Delta_{D_{0}}(t ; L)$ by Corollary 15. Secondly $\Delta_{D_{0} \cup L_{\infty}^{-d}}(t ; L)=\Delta_{D_{0} \cup L^{j}}(t)$ by Theorem 13. Lastly we have

$$
\sqrt{\Delta_{D_{0} \cup L_{\infty}^{-d}}(t ; L)}=\operatorname{gcd}\left(\Delta_{D_{0}}(t ; L) \times(t-1), t^{d}-1\right)
$$


The conclusion is now immediate from these observation, as the factor $(t-1)$ does not appear in $\Delta_{C_{\tau}}(t)$ by the irreducibility [24].

5.3.1. Examples of line degenerations of order 1. In 23, we have classified configurations of reduced sextics of torus type. Among them, there are sextics of torus type with components $B_{5}+L$ where $B_{5}$ is an irreducible quintic. In fact, each of them is a degeneration of irreducible sextics of torus type. We give one such example. $D_{0}=B_{5}$ has one $A_{11}$ singularity at $O=(0,0)$ and $L=\{Z=0\}$ and it is a flex tangent. A generic irreducible sextic $C_{t}$ has $\left[A_{11}+A_{5}\right]$ as singularities. By Theorem [14, we know that $\Delta_{C_{t}}(t ; L) \mid \Delta_{D_{0}}(t ; L)$. As $\Delta_{C_{t}}(t ; L)$ is divisible by the generic Alexander polynomial, which is $t^{2}-t+1$ by [22], we conclude $t^{2}-t+1 \mid \Delta_{D_{0}}(t ; L)$.

$$
\begin{gathered}
D_{0}: \quad x^{4} y-8 x^{2} y^{2}+7 y^{3}-4 x^{3} y-2 x^{2} y+y^{2}+x^{4}+2 x^{5} \\
+9 y^{4}-6 x^{3} y^{2}+2 y^{2} x+6 y^{3} x=0 \\
C_{s}:\left(-x^{2}+y\right)^{3}+\left(-x^{2}-x^{3}+y+3 y^{2}+x y+s x^{2} y\right)^{2}=0
\end{gathered}
$$

5.3.2. Line degenerations of order 2. We consider quartics which are non-reduced line degenerations of sextics. As a quartic $D$, we can take quartics with configuration (a) $\Sigma(D)=2 A_{2}$, or (b) $\Sigma(D)=2 A_{2}+A_{1}, 3 A_{2}$ (with an outer singularity $A_{1}$ or $A_{2}$ ) or (c) $\Sigma(D)=A_{5}$, $E_{6}$ or $\Sigma(D)=A_{2}+A_{3}, A_{6}$ (with a wild inner singularity).

We only explain here the case $(b)$ with $\Sigma(D)=3 A_{2}$. The other case will be explained systematically in $§ 5.4$. Three cuspidal quartic is very special. As the degenerated line, we can take any one of a simple tangent line or a tangent cone at a cusp or a unique bi-tangent line. This is not the case for other quartics listed above. The following family gives line degeneration of order 2 of sextics of torus type defined by $f_{2}^{3}+g_{3}^{2}=0$ into a quartic with 3 $A_{2}$ and a line $\{Z=0\}$ with multiplicity 2 .

(a-1) $L$ is a simple tangent line. $\left\{C_{s}, s \in \Delta\right\}$ is a family with an outer $A_{2}$ singularity at $Q=(0,1,0)$ and $L=\{Z=0\}$ is a simple tangent line of the quartic $D_{0}$. This degeneration is not a line-degeneration of torus curve which we study in the next section.

$$
\begin{gathered}
D_{0}: y^{4}-2 y^{2}+3 x y^{2}-3 / 4 x^{2} y^{2}+1-3 x+3 x^{2}-x^{3}=0 \\
C_{s}^{(1)}:\left(y-3 / 2 x y-y^{3}+s x^{3}\right)^{2}+\left(-y^{2}+1-x\right)^{3}=0
\end{gathered}
$$

(a-2) $L$ is a tangent cone of an $A_{2} \cdot\left\{C_{s}^{(2)}, s \in \Delta\right\}$ is a family with an $A_{1}$ singularity at $Q=(0,1,0)$ and $L=\{Z=0\}$ is a tangent cone of an $A_{2}$ singularity.

$$
\begin{gathered}
D_{0}:-3 x y^{3}+y^{4}+3 x y+1+3 x^{2} y^{2}-5 / 4 x^{3} y+3 / 16 x^{4}-3 / 4 x^{2}-2 y^{2}=0 \\
f_{2}(x, y, s)=1+(-1 / 4+s) x^{2}+x y-y^{2} \\
g_{3}(x, y, s)=-y^{3}+3 / 2 x y^{2}+\left(-3 / 4 x^{2}+1\right) y+1 / 8 x^{3}
\end{gathered}
$$

(a-3) $L$ is the bi-tangent line. As is observed in Proposition 11, the Alexander polynomial has multiplicity 2 for $\left(t^{2}-t+1\right)$. Thus this case is exceptional for the tangential Alexander polynomial of quartic with $3 A_{2}$. To expalin this we consider a family of sextics of torus type $\left\{C_{s}^{(3)}, s \in \Delta\right\}$ with $8 A_{2}$, where two $A_{2}$ are outer singularities and they are located at at 
$R=(0,5 / 2,1)$ and $R^{\prime}=(-2,1 / 2,1)$. An inner $A_{2}$ is at $Q=(0,7 / 2,1)$. The line $L=\{X=0\}$ is a bi-tangent line and the limit line degeneration. In an affine equation, we can define them as

$$
\begin{aligned}
& D_{0}: x y^{3}+\frac{1}{256}\left(19 x^{2}-12 y^{2}+75 x+75+6 x y\right)^{2}, C_{s}^{(3)}=\left\{f_{2}^{3}+g_{3}^{2}=0\right\} \\
& f_{2}(x, y, s)=\frac{1}{4(s-1)}\left(20 y s x+8 s^{2} x y-8 s^{3} x y-70 s+4 s^{3} x^{2}-20 s^{3} y-21 s^{2}+21 s^{3}-4 x y\right. \\
& \left.\quad-4 s^{2} x^{2}+20 s^{2} y-8 y^{2} s-4 s^{2} y^{2}+4 s^{3} y^{2}+48 y s-8 s x^{2}-48 s x+18 s^{3} x-18 s^{2} x\right) \\
& g_{3}(x, y, s)=\frac{1}{\left.16(s-1)^{2}\right)}\left(552 y s x+36 x y^{2} s^{5}-90 y s^{2} x^{2}+138 y s x^{2}-42 y s^{3} x^{2}+36 y^{2} s^{3} x-84 y^{2} x s^{4}\right. \\
& +36 y^{2} s^{2} x-108 y^{2} s x-336 s^{2} x y-96 s^{3} x y-75 x+516 x s^{4} y+120 x^{2} s^{4} y-228 s^{5} x y-48 s^{5} x^{2} y \\
& -525 s+24 y^{3} s+333 s^{5} x-75 x^{2}+117 s^{3} x^{2}+18 s^{3} y+420 s^{2}-161 s^{3}+21 s^{5} x^{3}-741 x s^{4} \\
& +12 x y^{2}-13 s x^{3}-518 s^{4}+259 s^{5}+16 s^{4} y^{3}-270 s^{5} y-354 s^{4} x^{2}+177 s^{2} x^{2}-288 s^{2} y \\
& \quad-8 s^{5} y^{3}-8 s^{3} y^{3}-168 s^{4} y^{2}-6 x^{2} y+540 s^{4} y-19 x^{3}+8 s^{2} x^{3}-204 y^{2} s+48 s^{2} y^{2} \\
& \left.+36 s^{3} y^{2}+144 s^{5} x^{2}+570 y s-213 s x^{2}-645 s x+15 s^{3} x+543 s^{2} x+36 s^{3} x^{3}-57 s^{4} x^{3}+84 y^{2} s^{5}\right)
\end{aligned}
$$

As the Alexander polynomial of sextics of torus type with $8 A_{2}$ is given $\left(t^{2}-t+1\right)^{2}([22]$, this explains $\Delta_{D_{0}}(t ; L)=\left(t^{2}-t+1\right)^{2}$. Four inner $A_{2}$ 's are not visible in the Figure 2, It is quite interesting to study how the family degenerates into $D_{0}+2 L$. Observe that two cusps of $D_{0}$ are not real points and $L=\{x=0\}$ is the bitangent line of $D_{0}$. Note that (a-1) and (a-2) can not be a line-degenerated torus curve in the sense of the next subsection.

FiguRE 2. $C_{s}^{(3)}, s=-1 / 3$, sextics with $8 A_{2}$ 
5.4. Line degeneration of curves of torus type. We consider a pair of coprime positive integers $p, q>1$ and consider the curves of $(p, q)$-torus curve:

$$
C_{p, q}: \quad f_{p}(X, Y, Z)^{q}+g_{q}(X, Y, Z)^{p}=0
$$

where $f_{p}, g_{q}$ are polynomials of degree $p, q$ respectively. Consider the special case that

$$
f_{p}(X, Y, Z)=f_{p-a}(X, Y, Z) \times Z^{a}, \quad g_{q}(X, Y, Z)=g_{q-b}(X, Y, Z) \times Z^{b}
$$

where $f_{p-a}, g_{q-b}$ are homogeneous polynomials of degree $p-a, q-b$ respectively and $0<a<p$ and $0<b<q$. Assume for example that $a q \geq b p$ and factoring $Z^{b p}$ from $f$, we have a curve

$$
D: \quad g(X, Y, Z)=f_{p-a}(X, Y, Z)^{q} Z^{q a-p b}+g_{q-b}(X, Y, Z)^{p}=0
$$

We call a curve $D$ a line-degenerated torus curve of type $(p, q)$ and we call the line $L=\{Z=0\}$ the limit line of the degeneration. Note that the degree of $D$ is $p q-b p$. The simplest case is $a=b=1$ and

$$
D: \quad g(X, Y, Z)=f_{p-1}(X, Y, Z)^{q} Z^{q-p}+g_{q-1}(X, Y, Z)^{p}=0, \quad p<q
$$

Theorem 18. For a degenerated torus curve $D$ of type $(p, q)$ defined (3), there is a family of line degeneration $f(X, Y, Z, t)$ of order bp such that $f_{0}(X, Y, Z)=g(X, Y, Z) Z^{\text {bp }}$ and each curve $C_{t}: f(X, Y, Z, t)=0$ passes through a fixed point $Q \in L$ and the multiplicity of $C_{t}$ at $Q$ is $p b$ for each $t \neq 0$.

Proof. We may assume that $Q=(0,1,0)$ is not on $D$. Let $h(X, Y)$ be a homogeneous polynomial of degree $q-b$ with $h(0, Y) \neq 0$. We put

$$
f_{p}(X, Y, Z)=f_{p-a}(X, Y, Z) Z^{a}, \quad g_{q}(X, Y, Z, t)=g_{q-b}(X, Y, Z) Z^{b}+t X^{b} h(X, Y)
$$

and put

$$
C_{t}: f(X, Y, Z, t):=f_{p}(X, Y, Z)^{q}+g_{q}(X, Y, Z, t)^{p}=0
$$

We can easily see that $C_{t}: f(X, Y, Z, t)=0$ passes through $Q$ and the multiplicity of $\left(C_{t}, Q\right)$ is $p b$, as the local equation at $Q$ is given by

$$
f\left(x^{\prime}, 1, z^{\prime}, t\right)=f_{p-a}\left(x^{\prime}, 1, z^{\prime}\right)^{q} z^{\prime q a}+\left(g_{q-b}\left(x^{\prime}, 1, z^{\prime}\right) z^{b}+t x^{\prime b} h\left(x^{\prime}, 1\right)\right)^{p}=0
$$

where $x^{\prime}=X / Y, z^{\prime}=Z / Y$. The affine equation of $C_{t}$ in $\mathbb{C}_{L}^{2}$ is given by

$$
f(x, y, 1, t)=f_{p-a}(x, y, 1)^{q}+\left(g_{q-b}(x, y, 1)+t x^{b} h(x, y)\right)^{p}=0 .
$$

where $x=X / Z, y=Y / Z$. We see that $\operatorname{degree}_{y} f(x, y, 1, t)=p q-p b$.

Put $s=\operatorname{gcd}(p, q)$. As the generic Alexander polynomial of $(p, q)$-torus curve of degree $p q$ is divisible by $\Delta_{p, q}(t):=\left(t^{p q / s}-1\right)^{s}(t-1) /\left(\left(t^{p}-1\right)\left(t^{q}-1\right)\right)$, we get

Corollary 19. Let $D$ be as above and let $L=\{Z=0\}$ be the limit line of the degeneration. Then $\Delta_{D}(t ; L)$ is divisible by $\Delta_{p, q}(t)$. 
5.4.1. Singularities of line-degenerated torus curves. We consider the curve defined by (3)

$$
D: \quad g(X, Y, Z)=f_{p-a}(X, Y, Z)^{q} Z^{q a-p b}+g_{q-b}(X, Y, Z)^{p}=0
$$

Suppose that $P \in D$ is a singular point. $P$ is called an inner singularity (respectively outer) if $f_{p-a}(P)=g_{q-b}(P)=0$ (resp. $\left.g_{q-b}(P) \neq 0\right)$. $P$ is called wild if $P$ is also on the limit line $Z=0$. The following describes the type of inner non-wild singularities.

Lemma 20. ([5]) Let $C$ be a curve of torus type

$$
C: \quad f(x, y)^{p}+g(x, y)^{q}=0, p<q
$$

and assume that $f(0,0)=g(0,0)=0$ and the curves $f(x, y)=0$ is smooth at $O$. Let $\nu$ be the local intersection number of $f(x, y)=g(x, y)=0$ at $O$. Then the singularity $(C, O)$ is topologically isomorphic to the Brieskorn singularity

$$
B_{p, q \nu}: y^{p}+x^{q \nu}=0
$$

The singularity is more complicated when $f(x, y)=0$ is singular at $O$. The description of wild singularities is also more complicated in general.

\subsection{Examples of line degeneration of torus curves.}

5.5.1. Cubic. A cuspidal cubic $Q$ can be understood as a line-degenerated (2,3)-torus curve of order 3 by taking

$$
\begin{gathered}
f_{2}(x, y)=y x, g_{3}(x, y)=g_{1}(x, y) x^{2} \\
Q: y^{3}+g_{1}(x, y)^{2} x=0 .
\end{gathered}
$$

The limit line of the degeneration is $L=\{x=0\}$. This explains that $\Delta_{Q}(t ; L)=t^{2}-t+1$.

5.5.2. Quartic. We give further quartics which can be a line-degenerated (2,3)-torus curve. We consider the quartics of the form:

$$
\begin{array}{cc}
C_{0}: & \left(f_{2}(x, y) x\right)^{2}+(y x)^{3}=0 \\
D: & g(x, y))=f_{2}(x, y)^{2}+y^{3} x=0
\end{array}
$$

where $f_{2}(x, y)$ is a polynomial of degree 2 and the limit line of degeneration is chosen to be $\{x=0\}$. In general, $D$ has two inner $A_{2}$ singularities at $y=f_{2}(x, y)=0$. If $y=0$ is tangent to the conic $C_{2}:=\left\{f_{2}(x, y)=0\right\}$, the singularity is an $A_{5}$. If moreover $C_{2}$ degenerates into two lines, the singularity is an $E_{6}$-singularity. The limit line $L$ is a bi-tangent line at $\{x=0\} \cap C_{2}$. If $L$ is tangent to the conic $C_{2}, D$ obtains a flex of order 2 and $L$ is the flex tangent line. Further more we can put one outer singularity, either $A_{1}$ or $A_{2}$. There are two more configurations which can be a line degeneration of torus curves: $A_{2}+A_{3}$ and $A_{6}$. For these singularities, we have to consider wild inner singularities. We have already studied most of these quartic and their Alexander invariants in 4.5 . Theorem 14 and Theorem 18 explains our previous computations. 
5.5.3. A quartic with $2 A_{2}$. For a generic quartic $D$ with $\Sigma(D)=2 A_{2}$, its dual curve $\check{D}$ is a sextic with $8 A_{2}+A_{1}$, in particular, $D$ has one bi-tangent line.

$$
D: g(x, y)=\left(y^{2}-1+x^{2}\right)^{2}+y^{3} x=0
$$

The bi-tangent line can degenerate into a flex tangent line of order 2 so that $\check{D}$ has $6 A_{2}+E_{6}$.

$$
D: g(x, y)=\left(y^{2}-2 y-2 x^{2}+x+1\right)^{2}+y^{3} x=0
$$

See Figure 4 $§ 5.9$ for graphs of these quartics.

5.5.4. A quartic with $2 A_{2}+A_{1}$. A generic quartic $D$ with $\Sigma(D)=2 A_{2}+A_{1}$ has two flexes and one bi-tangent line (i.e., the configuration space is self dual). Degenerated quartic $D^{\prime}$ has one flexes of order 2 and no bi-tangent line as we have seen before.

$$
\begin{gathered}
D:\left(y^{2}+\left(\frac{9}{2} x-1\right) y+\frac{3}{2} x^{2}+x-1\right)^{2}+y^{3} x=0 \\
D^{\prime}:\left(y^{2}+\left(\frac{17}{6} x-\frac{7}{3}\right) y+\frac{23}{6} x^{2}-\frac{13}{3} x+1\right)^{2}+y^{3} x=0
\end{gathered}
$$

See Figure 5. $§ 5.9$ for graphs of these quartics.

5.5.5. A quartic with $A_{5}$. A generic quartic $D$ with $\Sigma(D)=\left\{A_{5}\right\}$ has 6 flexes and one bitangent line, thus the dual curve is a sextic with $6 A_{2}+A_{5}+A_{1}$. Degenerated quartic $D^{\prime}$ has 4 flexes and one flex of order 2 and thus the dual curve is a sextic with $4 A_{2}+E_{6}+A_{5}$. Tokunaga has studied a certain dihedral covers branched along these quartics 33

$$
\begin{aligned}
D: & \left(y^{2}-y x-x^{2}+2 x-1\right)^{2}+y^{3} x=0 \\
D^{\prime}: & \left(y^{2}-2 y+x^{2}+2 x+1\right)^{2}+y^{3} x=0
\end{aligned}
$$

See Figure 6. §5.9 for graphs of these quartics.

5.5.6. A quartic with $E_{6}$. A generic quartic $D$ with $\Sigma(D)=\left\{E_{6}\right\}$ has two flexes and one bi-tangent line. Degenerated one $D^{\prime}$ has one flexes of order 2 and no bi-tangent line as we have seen before.

$$
D:\left(y^{2}-1-x^{2}+2 x\right)^{2}+y^{3} x=0 \quad D^{\prime}:(x-y+1)^{4}+y^{3} x=0
$$

Note that the last quartic $D^{\prime}$ with $E_{6}$ can be also considered as a line degeneration of $(3,4)$ torus curves as

$$
\left((x-y+1)^{4}+y^{3} x\right) x^{8}=\left((x-y+1) x^{2}\right)^{4}+\left(y x^{3}\right)^{3}
$$

This explains that $\Delta_{D^{\prime}}(t ; L)=\left(t^{2}-t+1\right)\left(t^{4}-t^{2}+1\right)$ with $L=\{x=0\}$. See Figure $7 \S 5.9$ for graphs of these quartics. 
5.5.7. quartics with $A_{2}+A_{3}$ and $A_{6}$. We start the general form

$$
C: f_{2}(x, y)^{2}+y^{3} x=0
$$

We assume that $O=(0,0)$ is a wild inner singularity. Thus $f_{2}(0,0)=0$.

The configuration $A_{2}+A_{3}$ is obtained when $f_{2}(0,0)=0$ and $f_{2}(x, y)=0$ is not tangent to $y=0$ as $(C, O) \cong A_{3}$. The limit line is tangent at one smooth point and also passing at $O$. For example,

$$
C:\left(-y^{2}+y-x^{2}+x\right)^{2}+y^{3} x=0
$$

If we make $f_{2}(x, y)=0$ is tangent to $y=0$ at $O,(C, O) \cong A_{6}$. An example is given as follows.

$$
C:\left(y^{2}+y+x^{2}\right)^{2}+y^{3} x=0
$$

See Figure 8, $\S 5.9$ for graphs of these quartics.

5.5.8. Quintics as line-degenerations. We consider (2,5)-torus curve $C: f(x, y)=f_{5}(x, y)^{2}+$ $g_{2}(x, y)^{5}=0$ of degree 10 which is degenerated as

$$
f_{5}(x, y)=f_{2}(x, y) x^{3}, g_{2}(x, y)=y x
$$

Then we get a quintic

$$
D: f_{2}(x, y)^{2} x+y^{5}=0
$$

In general, $D$ has $2 A_{4}$ singularities at $f_{2}(x, y)=y=0$ and it has a flex of order 3 at $O$ with the tangent line $L=\{x=0\}$. As a special case where the conic $f_{2}(x, y)=0$ is tangent to $y=0$, we get one $A_{9}$ singularity:

$$
D:\left(y^{2}+x y+x^{2}-2 x+1\right)^{2} x+y^{5}=0
$$

If $f_{2}(x, y)=0$ is two lines intersecting on $y=0$, the singularity is locally topologically isomorphic to $C_{5,5}$ in the notation [30]:

$$
C_{5,5}: y^{5}+x^{2} y^{2}+x^{5}=0
$$

If $f_{2}=0$ is a line with multiplicity 2 , the singularity becomes $B_{4,5}$ singularity which is locally defined as $y^{5}+x^{4}=0$.

There are two other possibilities. A quintic as a line degeneration of torus curves of type $(3,5)$ : take $g_{3}=y x^{2}, f_{5}=f_{1}(x, y) x^{4}$. Then we get a quintic

$$
Q: \quad y^{5}+x^{2} f_{1}(x, y)^{3}=0
$$

The quintic $Q$ has one $E_{8}$ singularity and a $A_{4}$ singularity on the limit line $x=0$. The limit line is also the tangent line of the singularity $A_{4}$.

Another possibility is as a line degeneration of torus curves of type $(4,5)$ : take $g_{4}=$ $y x^{3}, f_{5}=g_{1}(x, y) x^{4}$. Then we get a quintic

$$
Q^{\prime}: \quad y^{5}+x g_{1}(x, y)^{4}=0
$$

$Q^{\prime}$ has one $B_{5,4}$-singularity and the limit line is $x=0$. This quintic can be considered as a degeneration of (7) when $f_{2}(x, y) \rightarrow g_{1}(x, y)^{2}$. 
5.6. Sextics as line degenerations. Sextics as line degenerations can be either from $(2,5)$ torus curves, or from $(3,5)$-torus curves or from $(3,4)$-torus curves. The sextics from $(2,5)$-torus curves take the form:

$$
C: g(x, y)=f_{3}(x, y)^{2}+y^{5} x=0 .
$$

Generically $C$ has $3 A_{4}$ singularities and the degeneration line $L=\{x=0\}$ is a tri-tangent line. By the degeneration of the intersection $y=f_{3}(x, y)=0$, we may have also either $A_{4}+A_{9}$ or $A_{14}$. If the cubic $f_{3}(x, y)=0$ has a node or cusp, the singularity becomes more complicated. Sextics from $(3,5)$ torus curves take the form:

$$
C^{\prime}: f_{2}(x, y)^{3}+y^{5} x=0 .
$$

Generically $C^{\prime}$ has $2 B_{3,5}$ singularities and degeneration line is a bi-tangent line at two flex points. If $y=0$ is tangent to the conic $f_{2}(x, y)=0$, the singularity is $B_{3,10}$.

The curves $C: f_{2}(x, y)^{3}+y^{4} x^{2}=0$ can be considered as a line degeneration of $(3,4)$-torus curves but at the same time, it is a torus curve of type (2,3). Generically $C$ has $2 E_{6}+2 A_{2}$. Thus by Corollary [19] the Alexander polynomial $\Delta_{C}(t ; L)$ is divisible by $\left(t^{2}-t+1\right)\left(t^{4}-t^{2}+1\right)$. Note that $\Delta_{C}(t)=t^{2}-t+1$ by [27].

5.7. Flex degenerations. Let us consider $C_{\tau}$ is a family of irreducible curves in the configuration space $\mathcal{M}(\Sigma ; d)$ with two marked flex points $P_{\tau}, Q_{\tau} \in C_{\tau}$ of order 1 for $\tau \neq 0$ and assume that

(a) $P_{\tau}, Q_{\tau} \rightarrow P_{0}$ when $\tau \rightarrow 0$ and $P_{0}$ is a flex point of order 2 of $C_{0} \in \mathcal{M}(\Sigma ; d)$.

(b) The intersection $T_{P_{\tau}} C_{\tau} \cap T_{Q_{\tau}} C_{\tau} \cap C_{\tau}$ is empty for $\tau \neq 0$.

Theorem 21. Consider the degeneration: $C_{\tau}+L_{1, \tau}+L_{2, \tau} \rightarrow C_{0}+2 L$. Then we have the divisibility of Alexander polynomials:

$$
\Delta_{C_{\tau} \cup L_{1, \tau} \cup L_{2, \tau}}(t) \mid \Delta_{C_{0} \cup L^{2}}(t) \times(t-1) .
$$

Here $L_{1, \tau}=T_{P_{\tau}} C_{\tau}, L_{2, \tau}=T_{Q_{\tau}} C_{\tau}$ and $L=T_{P_{0}} C_{0}$.

Proof. First we may assume that $C_{\tau}=\{f(x, y, \tau)=0\}, P_{0}=(0,0)$ and the tangent line of $C_{0}$ at $P_{0}$ is defined by $y=0$. Take a generic line $L_{\infty}$ and we work in $\mathbb{C}^{2}=\mathbb{C}_{L_{\infty}}^{2}$. Taking a presenting polydisk $\Delta_{\alpha, \beta}$ for $C_{0} \cup L$ with pencil line $L_{\eta}, \eta \in \mathbb{C}$, let $\eta_{1}, \ldots, \eta_{m}$ be parameters corresponding to the singular pencil lines with $\eta_{1}=0$. Fix a small $\varepsilon>0$ to see the monodromy relations along $\left|\eta-\eta_{i}\right|=\varepsilon$. We take generators in a fixed generic line $L_{\eta_{0}},\left|\eta_{0}\right|=\varepsilon$ (see Appendix). Then we get a presentation:

$$
\pi_{1}\left(\mathbb{C}^{2}-C_{0} \cup L\right)=\left\langle g_{1}, \ldots, g_{d}, h \mid R_{1}, \ldots, R_{k}\right\rangle
$$

We consider the Alexander matrix $M_{0}$ with respect to the weight function $\theta_{2}$ which has weight 2 for $L$. Take a positive number $\delta$ so that

$$
C_{\tau} \pitchfork L_{\eta}, \quad \forall \tau,|\tau| \leq \delta, \forall \eta,\left|\eta-\eta_{i}\right|=\varepsilon, i=1, \ldots, m
$$

We may also assume that $\Delta_{\alpha, \beta}$ is admissible for $C_{\tau},|\tau| \leq \delta$. Next, we consider $C_{\tau} \cup L_{1, \tau} \cup L_{2, \tau}$ for sufficiently small $\tau$ in the above sense. In the generic fiber $L_{\eta}$, the intersection $L_{\eta} \cap L$ 
are bifurcated into two points $L_{\eta} \cap L_{1, \tau}$ and $L_{\eta} \cap L_{2, \tau}$, but they are observed only with a microscope and they move exactly as a twin satellite along $\left|\eta-\eta_{i}\right|=\varepsilon$. For the presentation of $\pi_{1}\left(\mathbb{C}^{2}-C_{\tau} \cup L_{2, \tau} \cup L_{2, \tau}\right)$, we need two generators $h_{1}, h_{2}$ presented by lassos for the lines $L_{2, \tau}$ and $L_{2, \tau}$ instead of one $h$. However we can understand as $h=h_{1} h_{2}$. For the further detail about the choice of generators, see Appendix. This implies that there are canonical homomorphisms $\psi, \Psi$ which make the next diagram commutative.

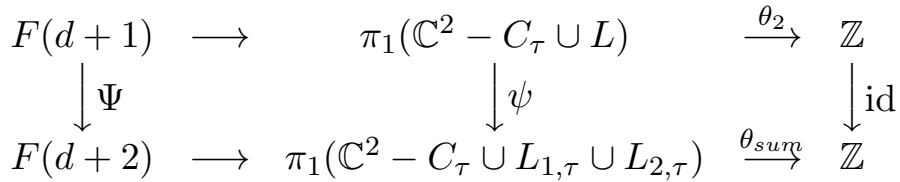

Here $\Psi$ is defined on generators as $g_{i} \mapsto g_{i}, h \mapsto h_{1} h_{2}$ and $\psi$ is canonically induced by $\Psi$. The monodromy relations $R_{1}, \ldots, R_{k}$ remains the same along $\left|\eta-\eta_{j}\right|=\varepsilon$. This means that the relation $R_{j}^{\prime}$ remains true where $R_{j}^{\prime}$ is obtained simply substituting the letter $h$ by $h_{1} h_{2}$. To get a complete relations, we have to add some more relations, say $S_{1}, \ldots, S_{\ell}$ along the singular pencil lines. Among them, we can assume that

$$
S_{1}=g_{d} h_{2} g_{d}^{-1} h_{2}^{-1}
$$

which is the relation at the transverse intersection $L_{2, \tau} \cap C_{\tau}$ near $P_{0}$. (In the appendix, we will explain this situation more.) Thus the presentation is given as

$$
\pi_{1}\left(\mathbb{C}^{2}-C_{\tau} \cup L_{1, \tau} \cup L_{2, \tau}\right)=\left\langle g_{1}, \ldots, g_{d}, h_{1}, h_{2} \mid R_{1}^{\prime}, \ldots, R_{k}^{\prime}, S_{1}, \ldots, S_{\ell}\right\rangle
$$

Let $G_{\infty}$ be the subgroup of $G_{\tau}:=\pi_{1}\left(\mathbb{C}^{2}-C_{\tau} \cup L_{1, \tau} \cup L_{2, \tau}\right)$ generated by $g_{1}, \ldots, g_{d}$ and the product $h_{1} h_{2}$. Then $\psi$ is a surjection on $G_{\infty} \subset G_{\tau}$. For $C_{\tau} \cup L_{1, \tau} \cup L_{2, \tau}$, we consider the summation homomorphism $\theta_{\text {sum }}$. Let $\gamma_{2}, \gamma_{\tau}$ be the ring homomorphisms corresponding to $\theta_{2}, \theta_{\text {sum }}$ :

$$
\gamma_{2}: \mathbb{C}(F(d+1)) \rightarrow \mathbb{C}\left[t, t^{-1}\right], \gamma_{\tau}: \mathbb{C}(F(d+2)) \rightarrow \mathbb{C}\left[t, t^{-1}\right]
$$

Note that the following diagrams are commutative.

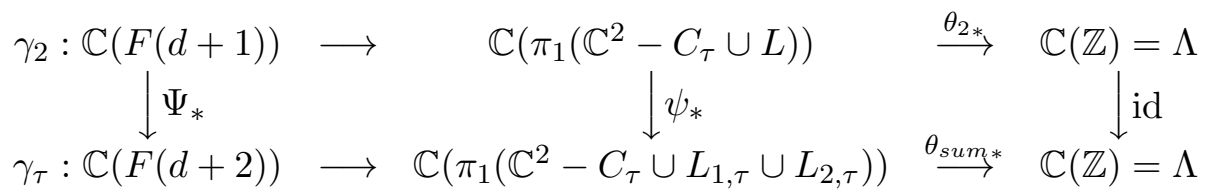

Now we consider the Alexander matrix $M_{\tau}$ of $C_{\tau} \cup L_{2, \tau} \cup L_{2, \tau}$. We consider the row corresponding to the relation $R_{i}^{\prime}$. It is a word of $g_{1}, \ldots, g_{d}$ and $h_{1} h_{2}$. By the definition of $\theta_{2}$, we can see easily that

$$
\gamma_{2}\left(\frac{\partial R_{i}}{\partial g_{j}}\right)=\gamma_{\tau}\left(\frac{\partial R_{i}^{\prime}}{\partial g_{j}}\right)
$$

The $(d+1)$-th column split into two columns, which correspond to the Fox differentials $\frac{\partial}{\partial h_{i}}, i=1,2$. As $h=h_{1} h_{2}$, we obtain

$$
\left(\gamma_{\tau}\left(\frac{\partial R_{i}^{\prime}}{\partial h_{1}}\right), \gamma_{\tau}\left(\frac{\partial R_{i}^{\prime}}{\partial h_{2}}\right)\right)=\left(\gamma_{2}\left(\frac{\partial R_{i}}{\partial h}\right), t \times \gamma_{2}\left(\frac{\partial R_{i}}{\partial h}\right)\right.
$$

Note also that

$$
\left(\gamma_{\tau}\left(\frac{\partial S_{1}}{\partial h_{1}}\right), \gamma_{\tau}\left(\frac{\partial S_{1}}{\partial h_{2}}\right)\right)=(0, t-1)
$$


Let $M_{\tau}^{\prime}$ be the matrix obtained by adding $(-t) \times(d+1)$-th column to $(d+2)$-th column so that the last column is zero up to $k$-th row. $M_{\tau}^{\prime}$ is written as

$$
\left(\begin{array}{cc}
M_{0} & \overrightarrow{0} \\
\mathbf{w} & (t-1) \\
N_{1} & \vec{v}
\end{array}\right)
$$

where $M_{0}$ is the Alexander matrix of $C_{0} \cup L$ and $\mathbf{w}$ comes from the differential of $S_{1}$, and the other terms $N_{1}, \vec{v}$ are coming from $S_{j}, j \geq 2$. Thus for any $(d-1) \times(d-1)$-minor $A$ of $M_{0}$, we associate $d \times d$-minor $A^{\prime}$ of $M_{\tau}^{\prime}$, by adding $(k+1)$-row and the last column. Then the corresponding determinant is equal to $\operatorname{det}(A) \times(t-1)$. Thus the assertion follows from the Fox calculus definition of the Alexander polynomial.

5.8. Appendix. In this appendix, we will explain the existence of the relation $S_{1}$ in the proof of Theorem 21. First we may assume that $C_{\tau}=\{f(x, y, \tau)=0\}, P_{0}=(0,0)$ and the tangent line of $C_{0}$ at $P_{0}$ is defined by $y=0$. Changing the scale and using Implicit function theorem, we may assume that $C_{0}$ is defined by $y=\phi_{0}(x)$ where $\phi_{0}(x)=x^{4}+$ higher terms in the polydisk $\Delta_{1,1}=\{(x, y) ;|x|,|y| \leq 1\}$. This follows from the assumption that $O$ is a flex of order 2 of $C_{0}$. We consider the pencil lines $x=\eta$. Now we consider $C_{\tau}$. Assume that $C_{\tau}$ is defined by $y=\phi_{\tau}(x)$ in $\Delta_{1,1}$. Write $\phi_{\tau}(x)=\sum_{\nu} c_{\nu}(\tau) x^{\nu}$. First observe that $\left|c_{4}(\tau)-1\right| \ll 1$ by continuity. In the parametrization, the flex points are defined by $\left\{(\alpha, \beta) \mid \phi_{\tau}^{\prime \prime}(\alpha)=0\right\}$. Thus by Rouché's principle, we see that there is two flex points which bifurcate from $P_{0}$. They corresponds to the roots of $\phi_{\tau}^{\prime \prime}(x)=0$ in $|x| \leq 1$, say $x=\alpha_{1}(\tau), \alpha_{2}(\tau)$. Thus $L_{1, \tau}, L_{2, \tau}$ corresponds to the tangent line at these flex points. By Bézout's theorem and the local stability of intersection numbers, there is one transverse intersection point of $L_{i, \tau} \cap C_{\tau}$ and we put them $Q_{i}=\left(\beta_{i}, \phi_{\tau}\left(\beta_{i}\right)\right)$ for $i=1,2$. Note that $\beta_{i} \rightarrow 0$ when $\tau \rightarrow 0$. Thus the local singular pencils for $\widetilde{C}_{\tau}=C_{\tau} \cup L_{1, \tau} \cup L_{2, \tau}$ is bifurcated in four points $x=\alpha_{i}, \beta_{i}, i=1,2$. We consider the local geometry of $p:\left(\Delta_{1,1}, \Delta_{1,1} \cap \widetilde{C}_{\tau}\right) \rightarrow \Delta$. Let $\check{\phi}_{\tau}(x)$ be the polynomial of degree 4 which is the Taylor expansion of $\Phi_{\tau}(x)$ modulo $x^{5}$. First we observe that this branched covering $p:\left(\Delta_{1,1}, \Delta_{1,1} \cap \widetilde{C}_{\tau}\right) \rightarrow \Delta$ is topologically equivalent to the one where we replace $C_{\tau}$ by the curve $\check{C}_{\tau}=\left\{y=\check{\phi}_{\tau}(x)\right\}$. Next, the situation for $\check{C}_{\tau}$ and its two flex tangents inside $\Delta_{1,1}$ is equivalent to the following explicit one:

$$
C_{\tau}^{\prime}: y=\xi_{\tau}(x), \quad \xi_{\tau}(x):=x^{4}-6 \tau^{2} x^{2}
$$

For this, we consider simply a homotopy $\Xi_{t}(x)=t \xi_{\tau}(x)+(1-t) \check{\phi}_{\tau}(x)$. Except a finite number of $t=t_{1}, \ldots, t_{q}$, this family of curves defines equivalent covering over $\Delta$. In this model $\xi_{\tau}(x)$, we have $\alpha_{1}, \alpha_{2}= \pm \tau$ and $\beta_{1}, \beta_{2}=\mp 3 \tau$. We choose $\{x=1\}$ as the fixed generic fiber i.e., $\eta_{0}=1$. On the fiber $x=1, P, Q_{1}, Q_{2}$ are the intersections of the line $x=1$ and $C, L_{1}, L_{2}$ respectively and we choose the generators as in Figure 7. The base point $B$ is chosen on the circle $|y|=1$. The other $d-1$ intersection points of $C_{\tau} \cap\{x=1\}$ are outside of the unit disk and the generators $g_{1}, \ldots, g_{d-1}$ are omitted in the figure. The loops are oriented counterclockwise. Now we consider the loop in the base space $\ell \circ \omega \circ \ell^{-1}$ where $\ell$ is the line segment from $x=1$ to $x=\beta_{2}+\varepsilon^{\prime}, \varepsilon^{\prime}<<\left(1-\beta_{2}\right)$ and $\omega$ is the loop $\left|\eta-\beta_{2}\right|=\varepsilon^{\prime}$. It is now 
easy to see that the monodromy relation along this loop is nothing but $S_{1}: g_{d} h_{2} g_{d}^{-1} h_{2}^{-1}$ as is expected.

Figure 3. Choice of generators $g_{d}, h_{1}, h_{2}$

5.9. Graphs of various quartics. We put the graphs of various line-degenerated quartics of torus type.

FIgURE 4. Quartic with $2 A_{2}$, bi-tangent limit line (left), flex tangent limit line (right) 
FiguRE 5. Quartic with $2 A_{2}+A_{1}$, bi-tangent limit line (left), flex tangent limit line (right)

Figure 6. Quartic with $A_{5}$, bi-tangent limit line (left), flex tangent limit line (right)

Figure 7. Quartic with $E_{6}$, bi-tangent limit line (left), flex tangent limit line (right) 
Figure 8. Quartic with $A_{3}+A_{2}$ left, with $A_{6}$ right

\section{REFERENCES}

[1] E. Artal Bartolo. Sur les couples des Zariski. J. Algebraic Geometry, 3:223-247, 1994.

[2] E. Artal Bartolo and J. Carmona Ruber. Zariski pairs, fundamental groups and Alexander polynomials. J. Math. Soc. Japan, 50(3):521-543, 1998.

[3] E. Artal Bartolo and H.-o. Tokunaga. Zariski pairs of index 19 and Mordell-Weil groups of K3 surfaces. Proc. London Math. Soc. (3), 80(1):127-144, 2000.

[4] E. Artin. Theory of braids. Ann. of Math. (2), 48:101-126, 1947.

[5] B. Audoubert, C. Nguyen, and M. Oka. On alexander polynomials of torus curves. J. Math. Soc. Japan, 57(4):935-957, 2005.

[6] R. H. Crowell and R. H. Fox. Introduction to Knot Theory. Ginn and Co., Boston, Mass., 1963.

[7] A. I. Degtyarev. Alexander polynomial of a curve of degree six. J. Knot Theory Ramifications, 3:439-454, 1994.

[8] P. Deligne. Le groupe fondamental du complément d'une courbe plane n'ayant que des points doubles ordinaires est abélien (d'après W. Fulton). In Bourbaki Seminar, Vol. 1979/80, volume 842 of Lecture Notes in Math., pages 1-10. Springer, Berlin, 1981.

[9] W. Fulton. On the fundamental group of the complement of a node curve. Ann. of Math. (2), 111(2):407409, 1980.

[10] J. Harris. On the Severi problem. Invent. Math., 84(3):445-461, 1986.

[11] A. Libgober. Alexander polynomial of plane algebraic curves and cyclic multiple planes. Duke Math. J., 49(4):833-851, 1982.

[12] A. Libgober. Fundamental groups of the complements to plane singular curves. In Algebraic geometry, Bowdoin, 1985, volume 46, Part II of Proc. Symp. Pure Math., pages 29-45. Amer. Math. Soc., Providence, RI, 1987.

[13] A. Libgober. Characteristic varieties of algebraic curves. In Applications of algebraic geometry to coding theory, physics and computation (Eilat, 2001), volume 36 of NATO Sci. Ser. II Math. Phys. Chem., pages 215-254. Kluwer Acad. Publ., Dordrecht, 2001.

[14] F. Loeser and M. Vaquié. Le polynôme d'Alexander d'une courbe plane projective. Topology, 29(2):163$173,1990$.

[15] M. Namba. Geometry of projective algebraic curves. Decker, New York, 1984.

[16] M. V. Nori. Zariski's conjecture and related problems. Ann. Sci. École Norm. Sup. (4), 16(2):305-344, 1983. 
[17] M. Oka. The monodromy of a curve with ordinary double points. Invent. Math., 27:157-164, 1974.

[18] M. Oka. On the fundamental group of the complement of certain plane curves. J. Math. Soc. Japan, 30(4):579-597, 1978.

[19] M. Oka. Symmetric plane curves with nodes and cusps. J. Math. Soc. Japan, 44(3):376-414, 1992.

[20] M. Oka. Two transforms of plane curves and their fundamental groups. J. Math. Sci. Univ. Tokyo, 3:399443, 1996.

[21] M. Oka. Geometry of cuspidal sextics and their dual curves. In Singularities — Sapporo 1998, pages $245-277$. Kinokuniya, Tokyo, 2000.

[22] M. Oka. Alexander polynomial of sextics. J. Knot Theory Ramifications, 12(5):619-636, 2003.

[23] M. Oka. Geometry of reduced sextics of torus type. Tokyo J. Math., 26(2):301-327, 2003.

[24] M. Oka. A survey on Alexander polynomials of plane curves. Singularités Franco-Japonaise, Séminaire et congrès, 10:209-232, 2005.

[25] M. Oka. Zariski pairs on sextics I. math.AG/0507051, 2005.

[26] M. Oka. Zariski pairs on sextics II. math.AG/0507052 2005.

[27] M. Oka and D. Pho. Fundamental group of sextic of torus type. In Trends in Singularities, pages 151-180. Birkhäuser, Basel, 2002.

[28] M. Oka and K. Sakamoto. Product theorem of the fundamental group of a reducible curve. J. Math. Soc. Japan, 30(4):599-602, 1978.

[29] S. Y. Orevkov. The commutant of the fundamental group of the complement of a plane algebraic curve. Uspekhi Mat. Nauk, 45(1(271)):183-184, 1990.

[30] D. T. Pho. Classification of singularities on torus curves of type (2,3). Kodai Math. J., 24(2):259-284, 2001.

[31] R. Randell. Milnor fibers and Alexander polynomials of plane curves. In Singularities, Part 2 (Arcata, Calif., 1981), pages 415-419. Amer. Math. Soc., Providence, RI, 1983.

[32] I. Shimada. Fundamental groups of complements to singular plane curves. Amer. J. Math., 119(1):127-157, 1997.

[33] H.-o. Tokunaga. Dihedral covers and an elementary arithmetic on elliptic surfaces. J. Math. Kyoto Univ., 44(2):255-270, 2004.

[34] H. H. Vui and L. D. Tráng. Sur la topologie des polynômes complexes. Acta Math. Vietnam., 9(1):21-32 (1985), 1984.

[35] C. Wall. Geometry of quartic curves. Math. Proc. Camb. Phil. Soc., 117:415-423, 1995.

[36] O. Zariski. On the problem of existence of algebraic functions of two variables possessing a given branch curve. Amer. J. Math., 51:305-328, 1929.

\section{Department of Mathematics}

Tokyo University of SCIENCE

26 WAKAMIYA-CHO, SHINJUKU-KU

TOKYO 162-8601

E-mail: oka@rs.kagu.tus.ac.jp 


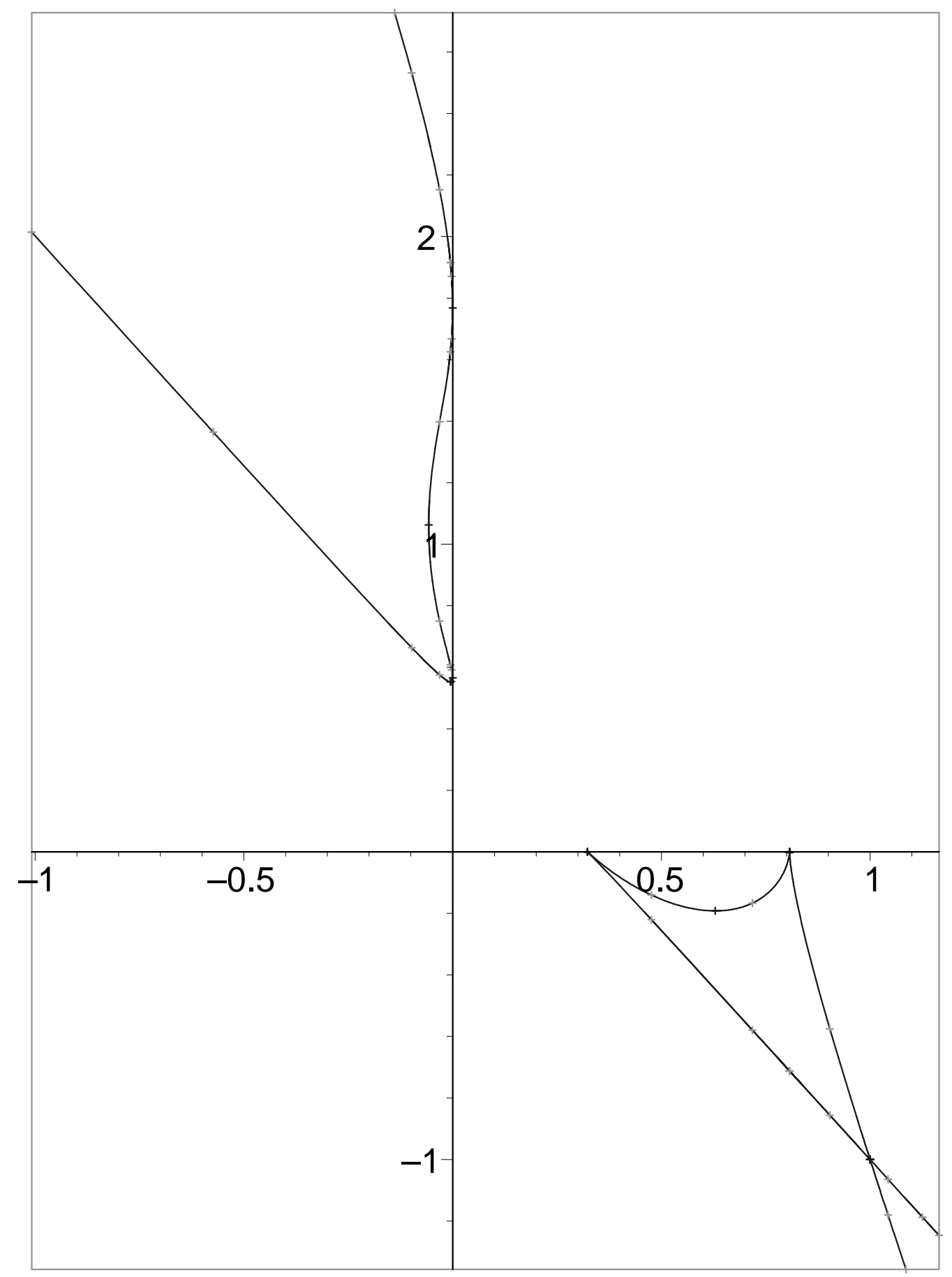




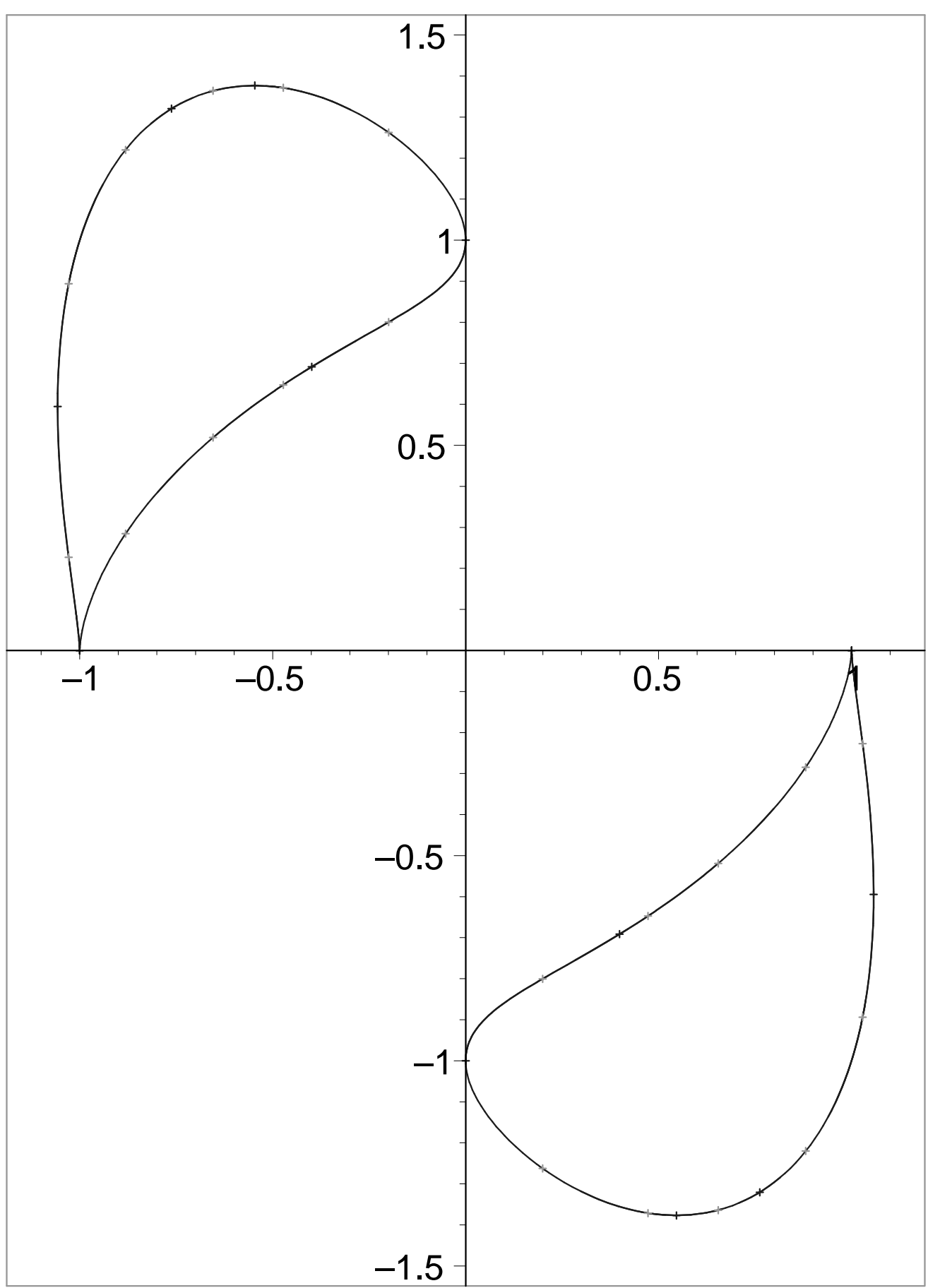




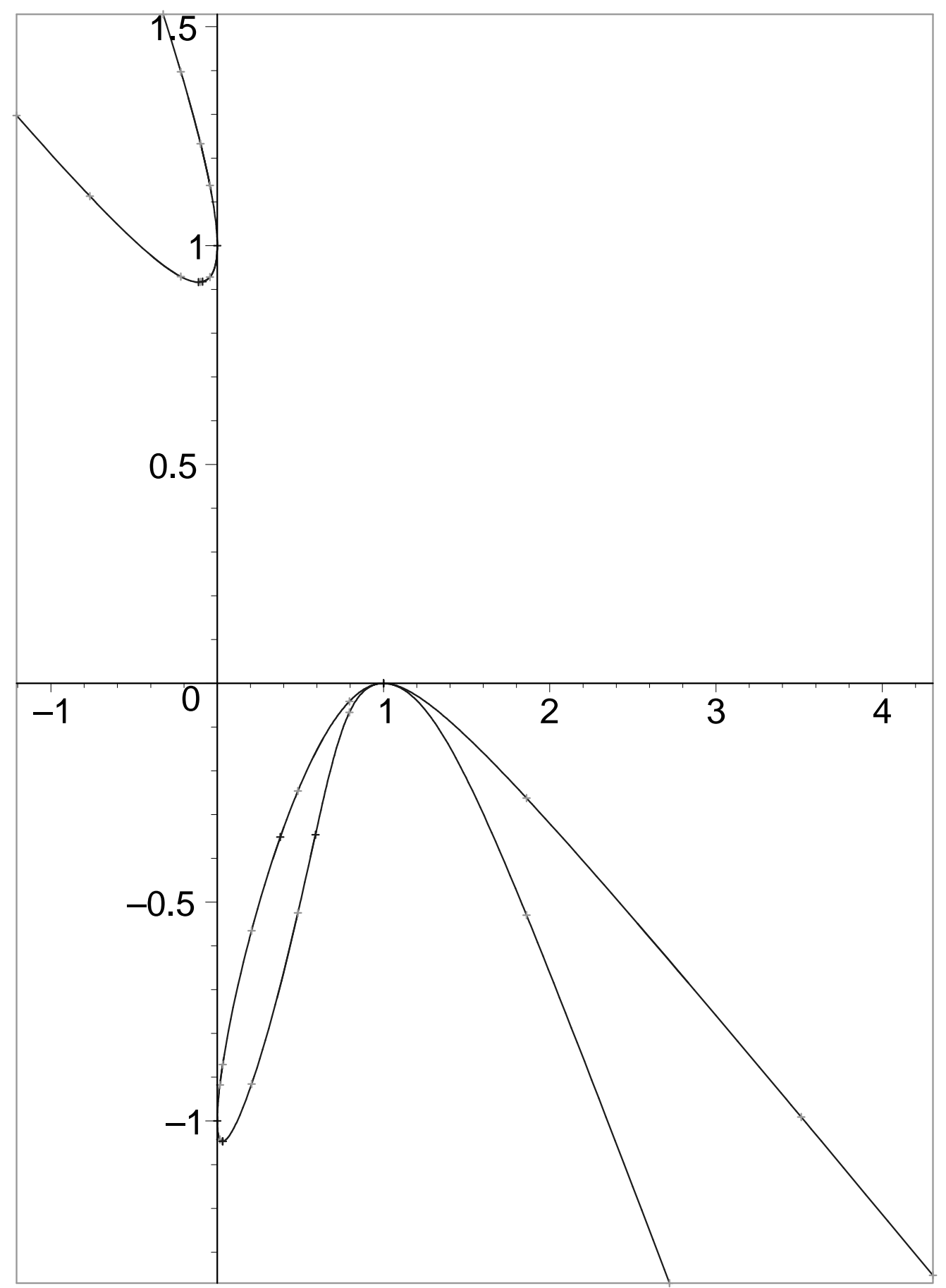




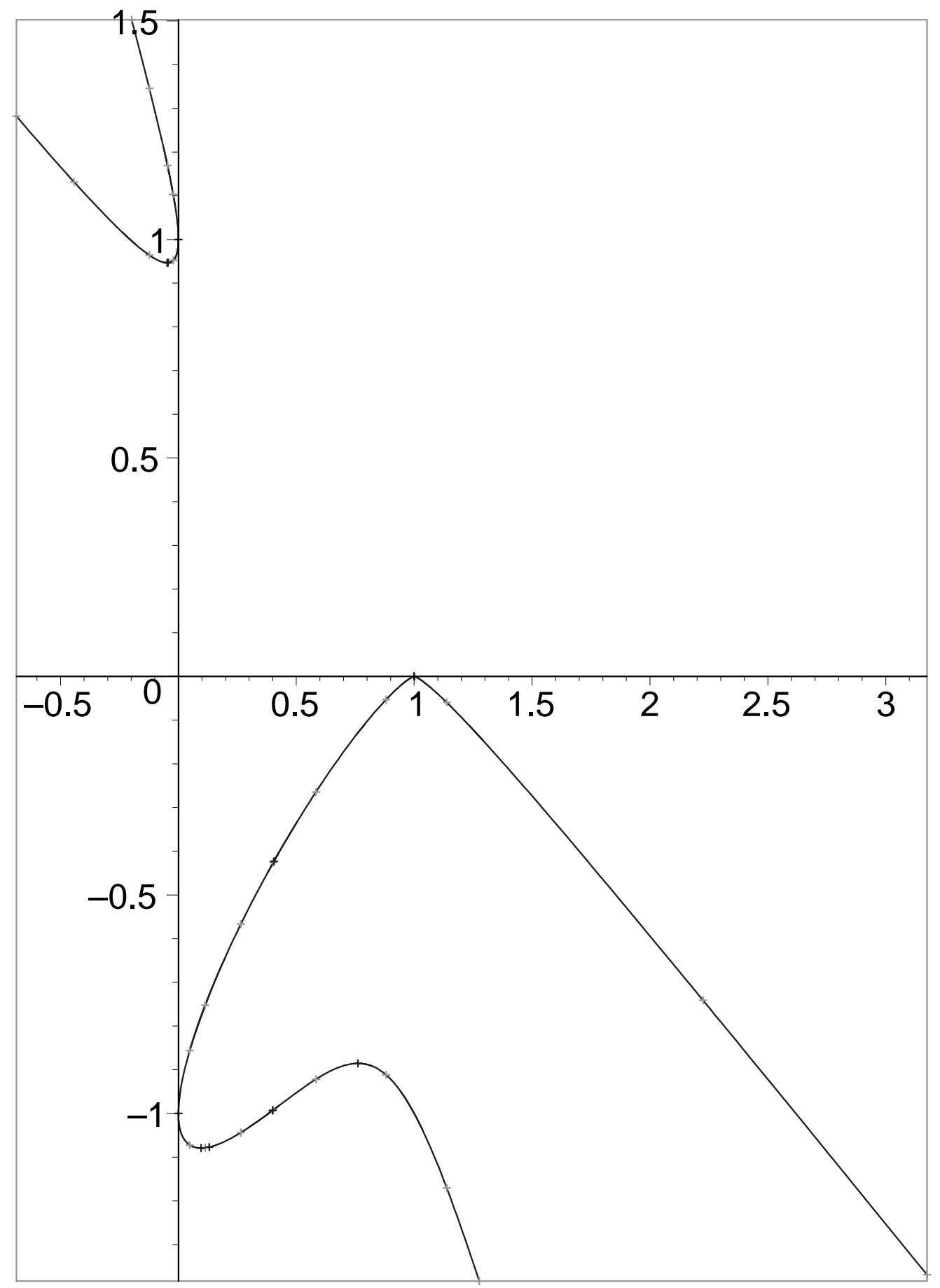




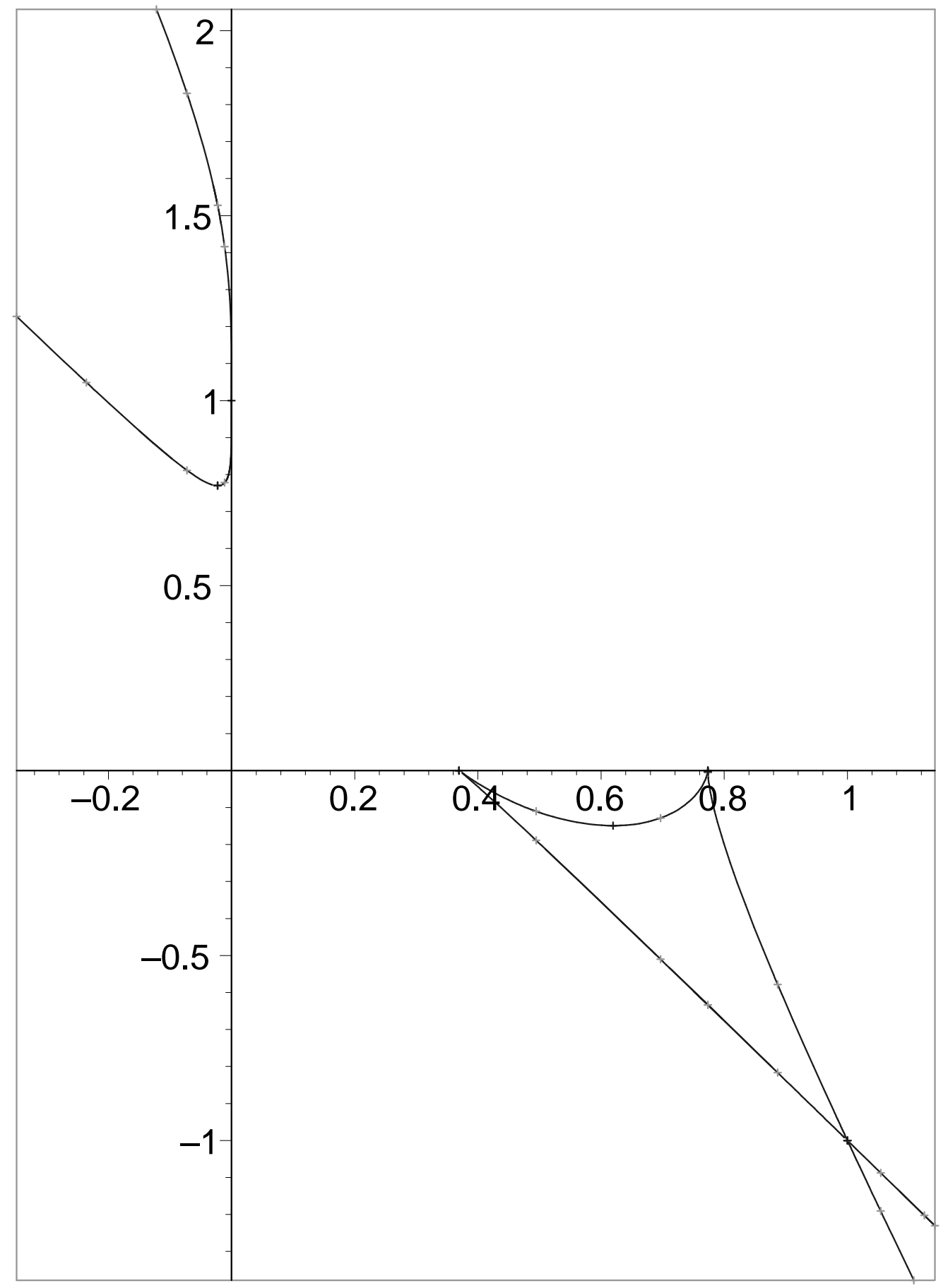




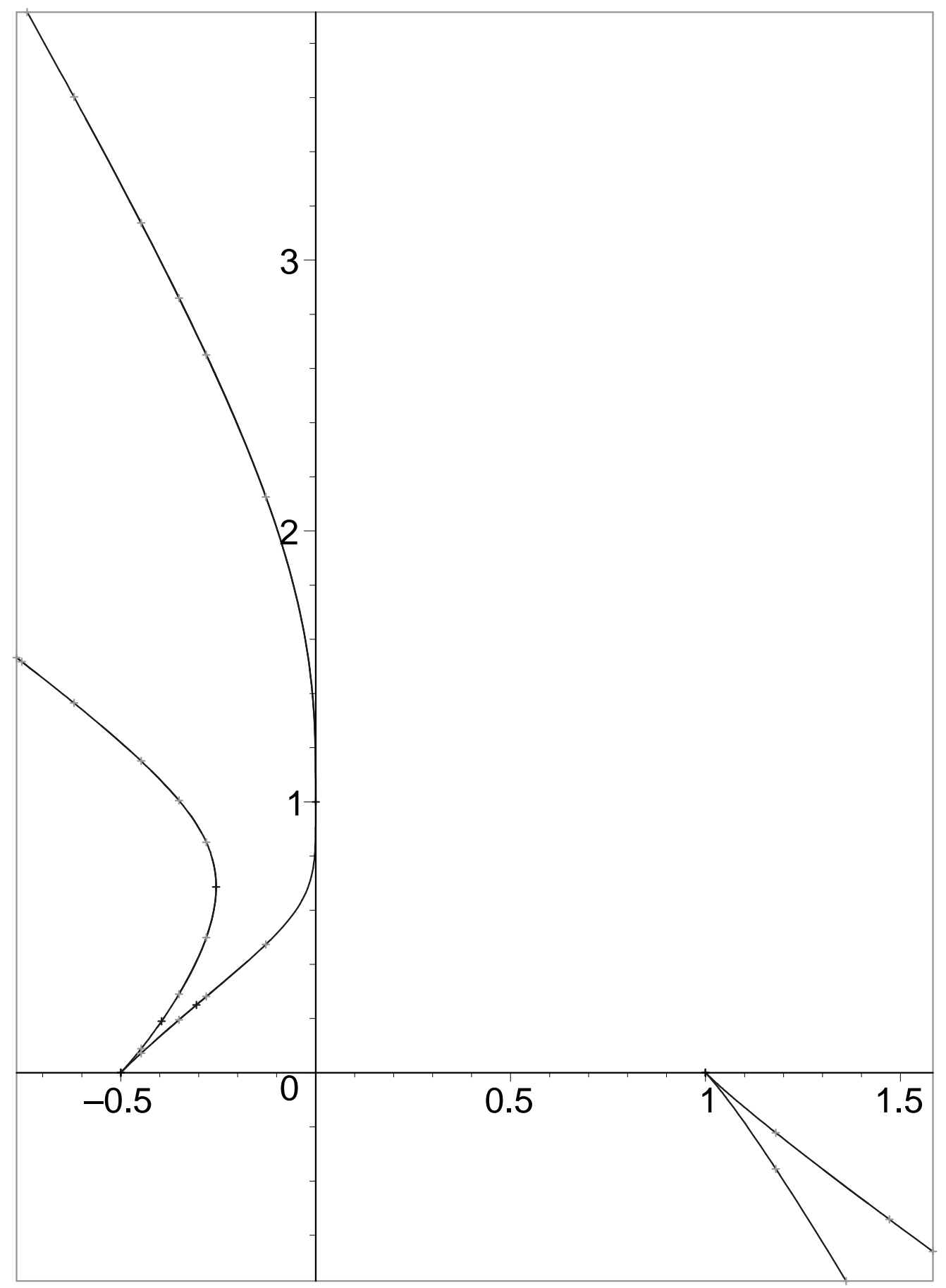




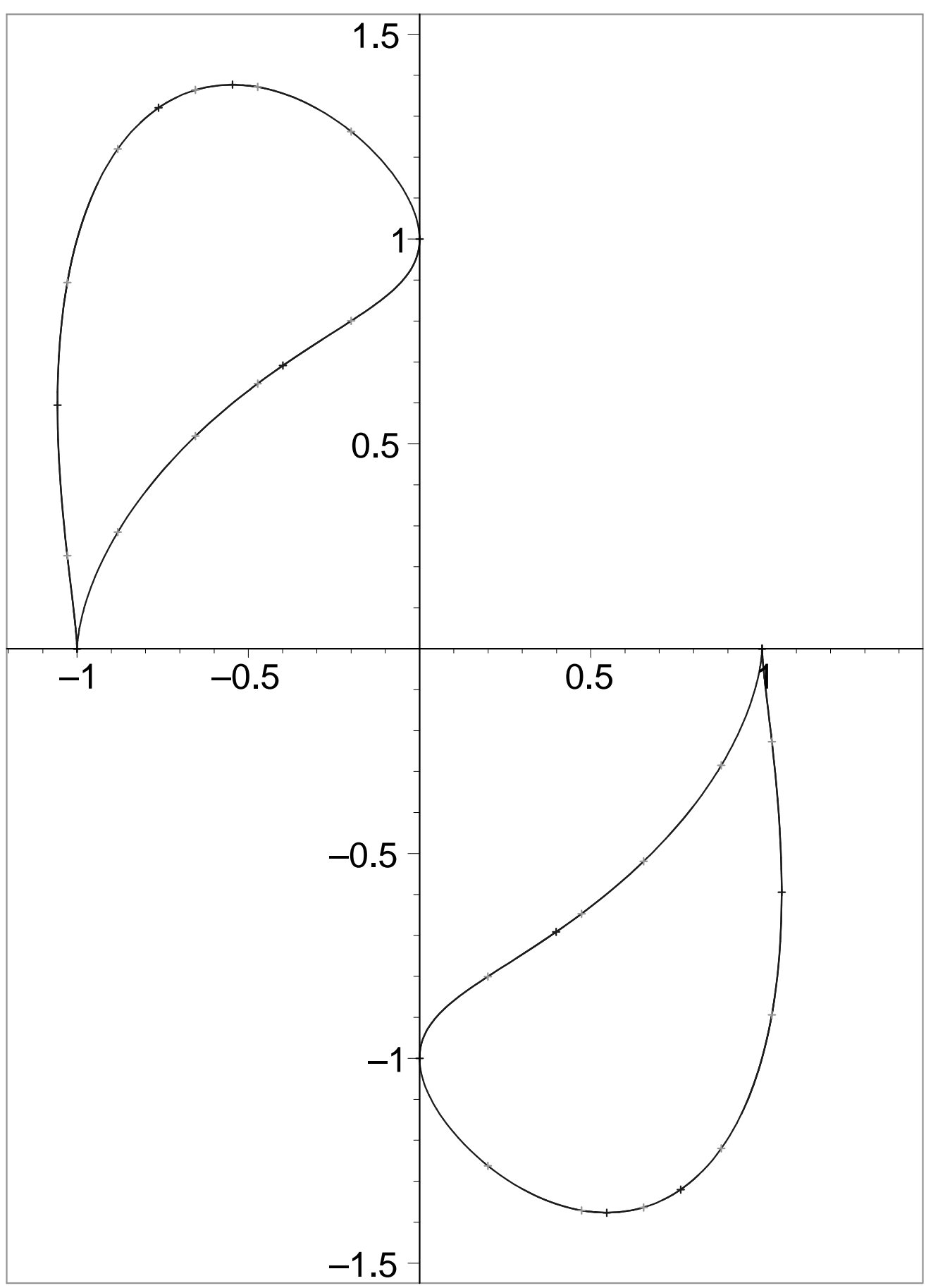




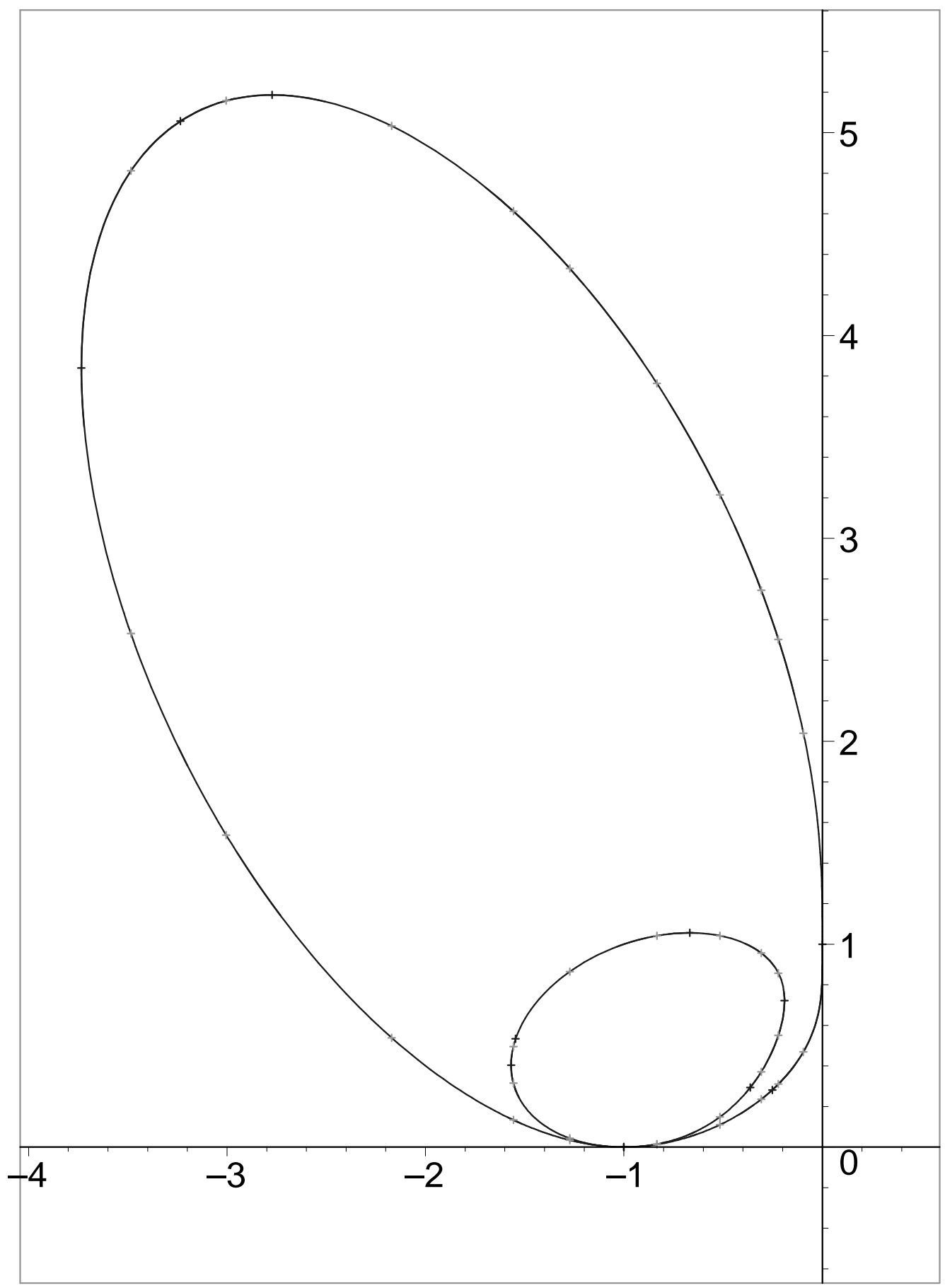




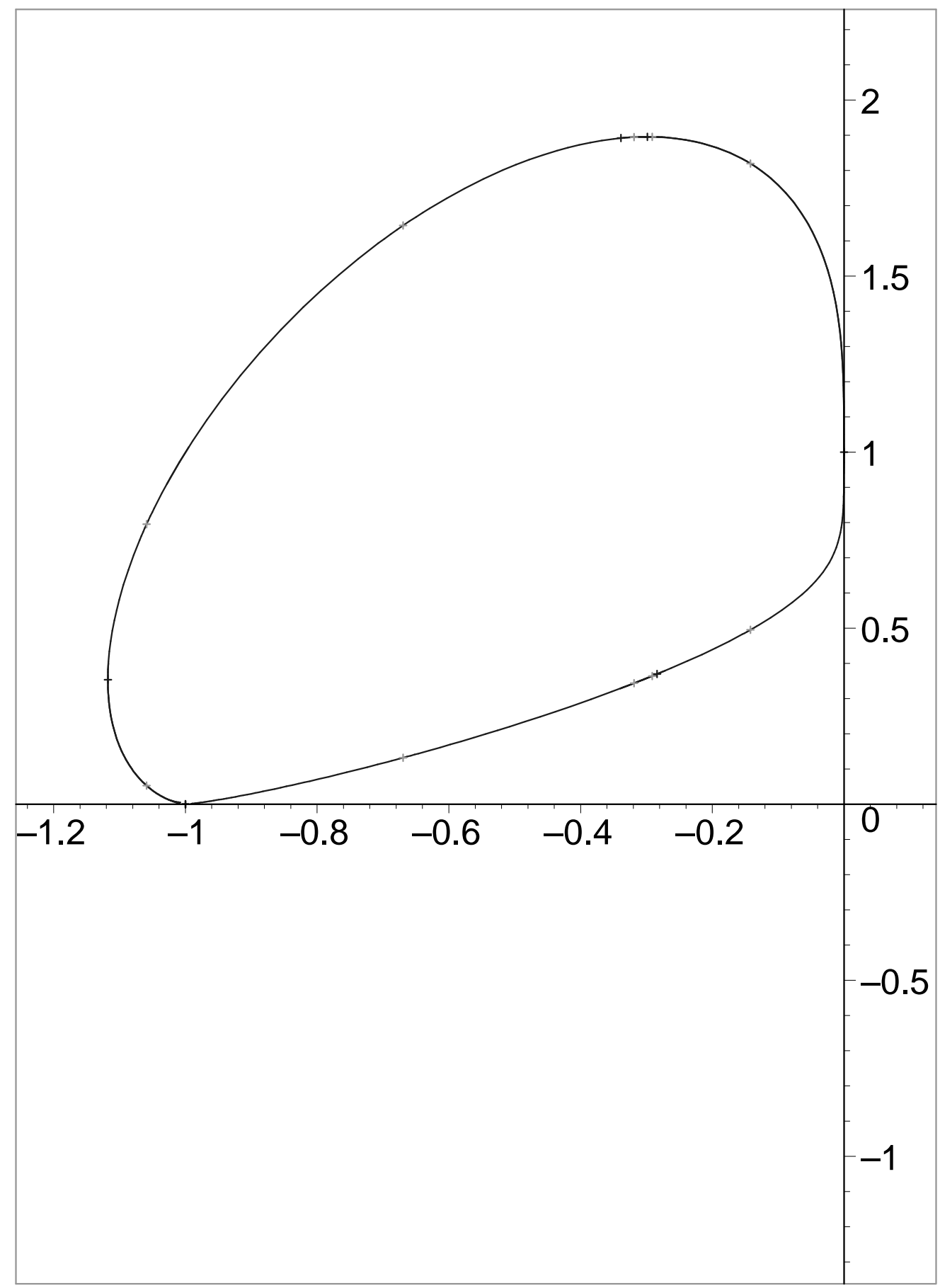




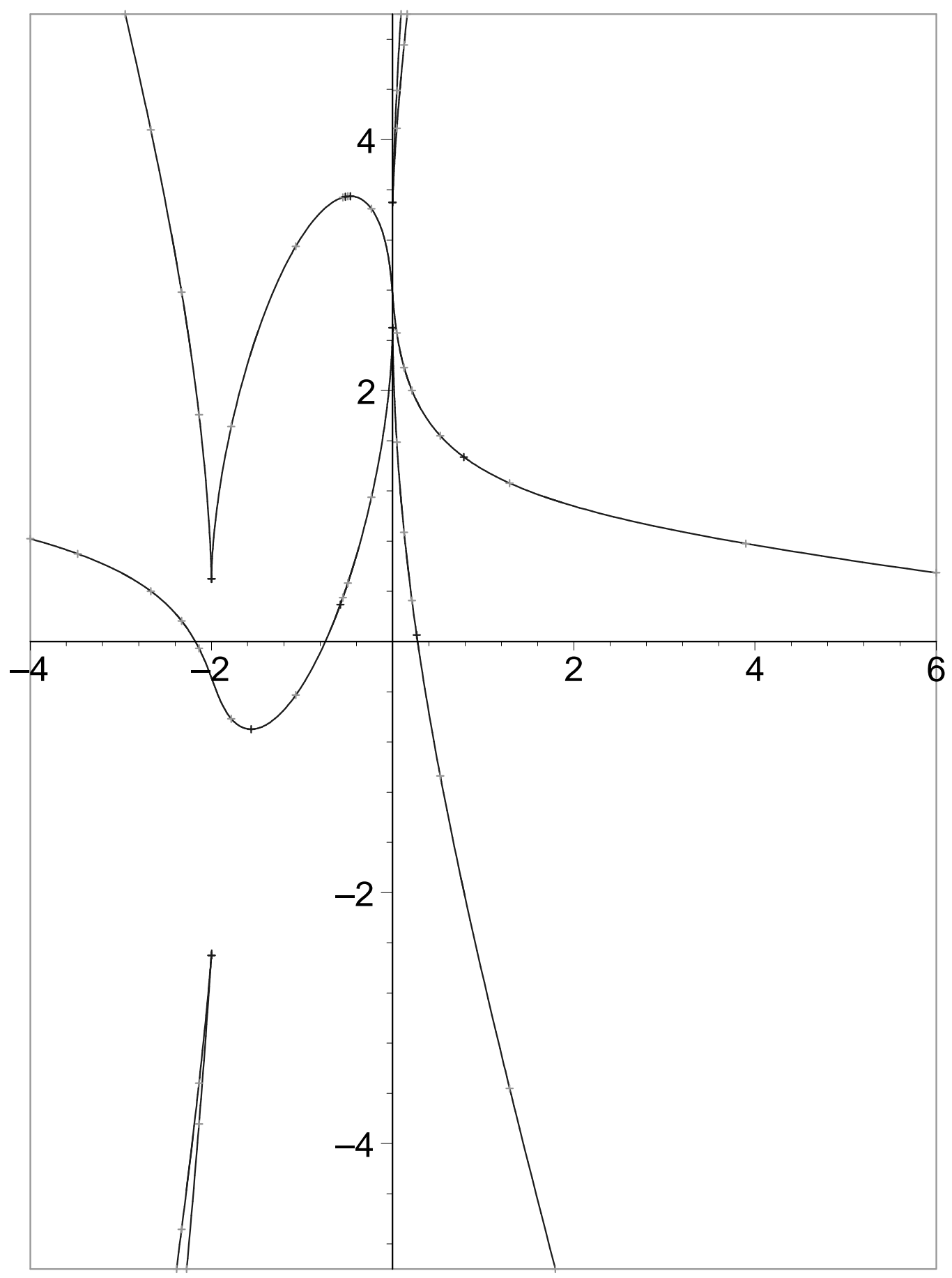




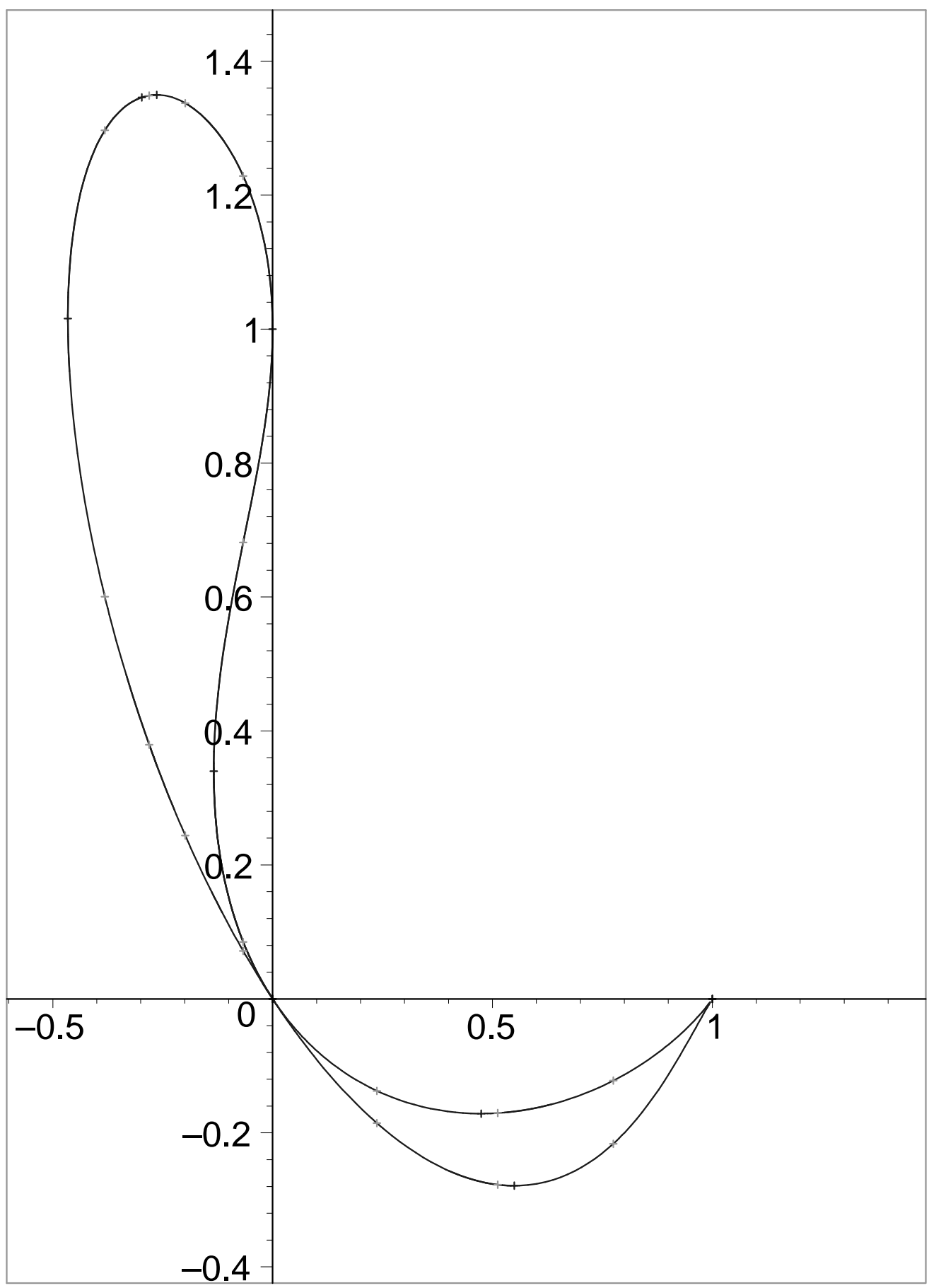




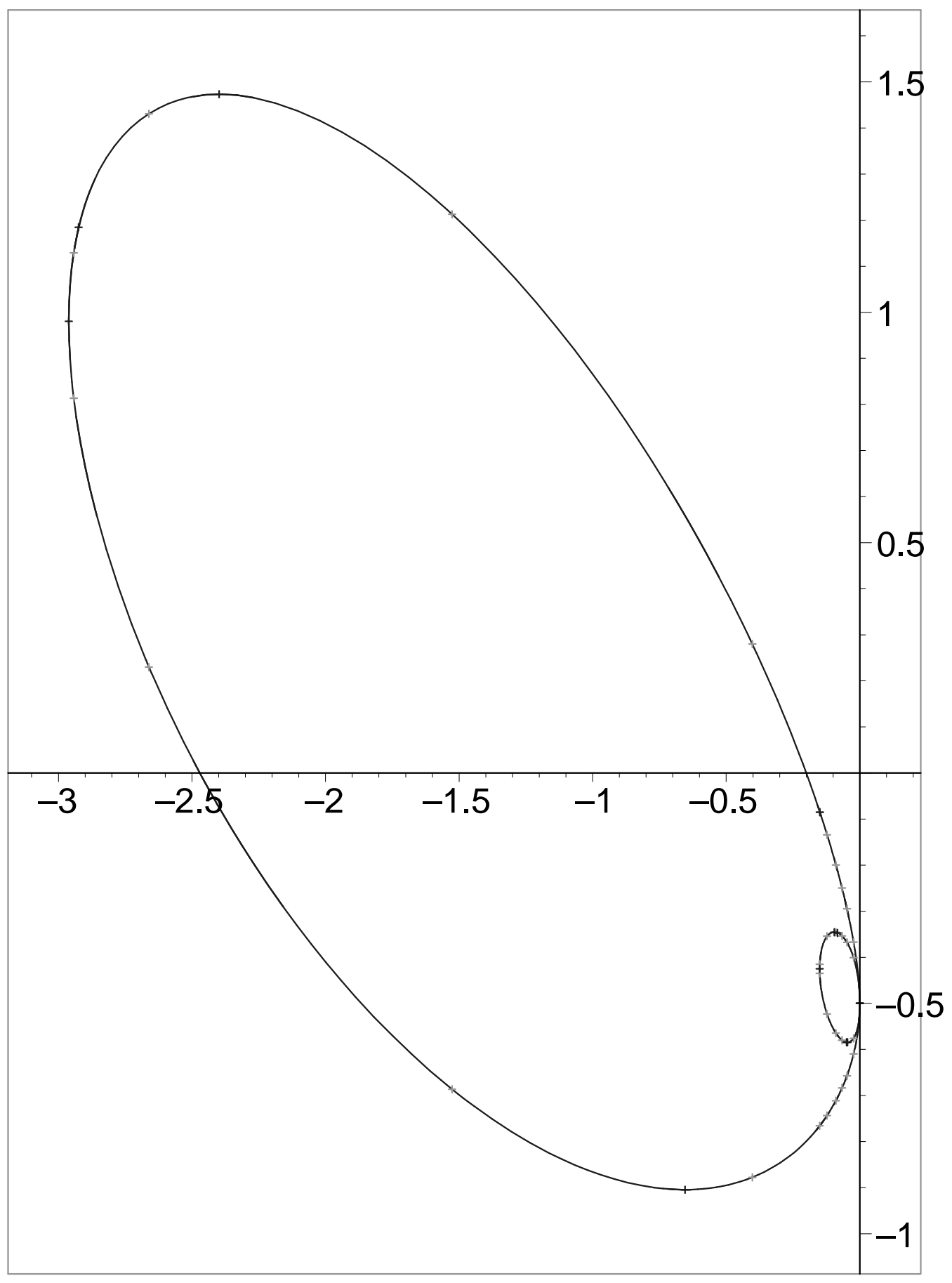




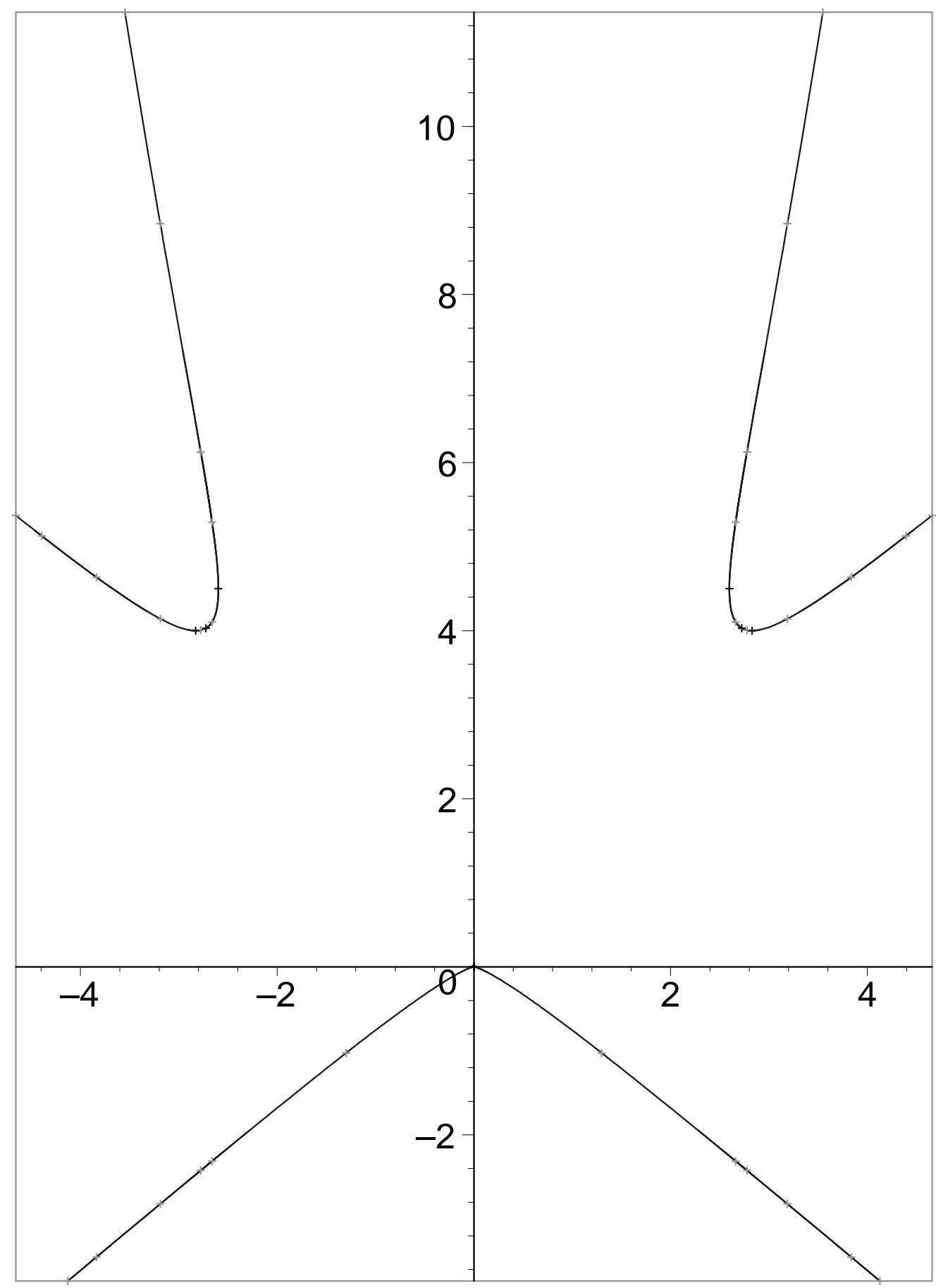




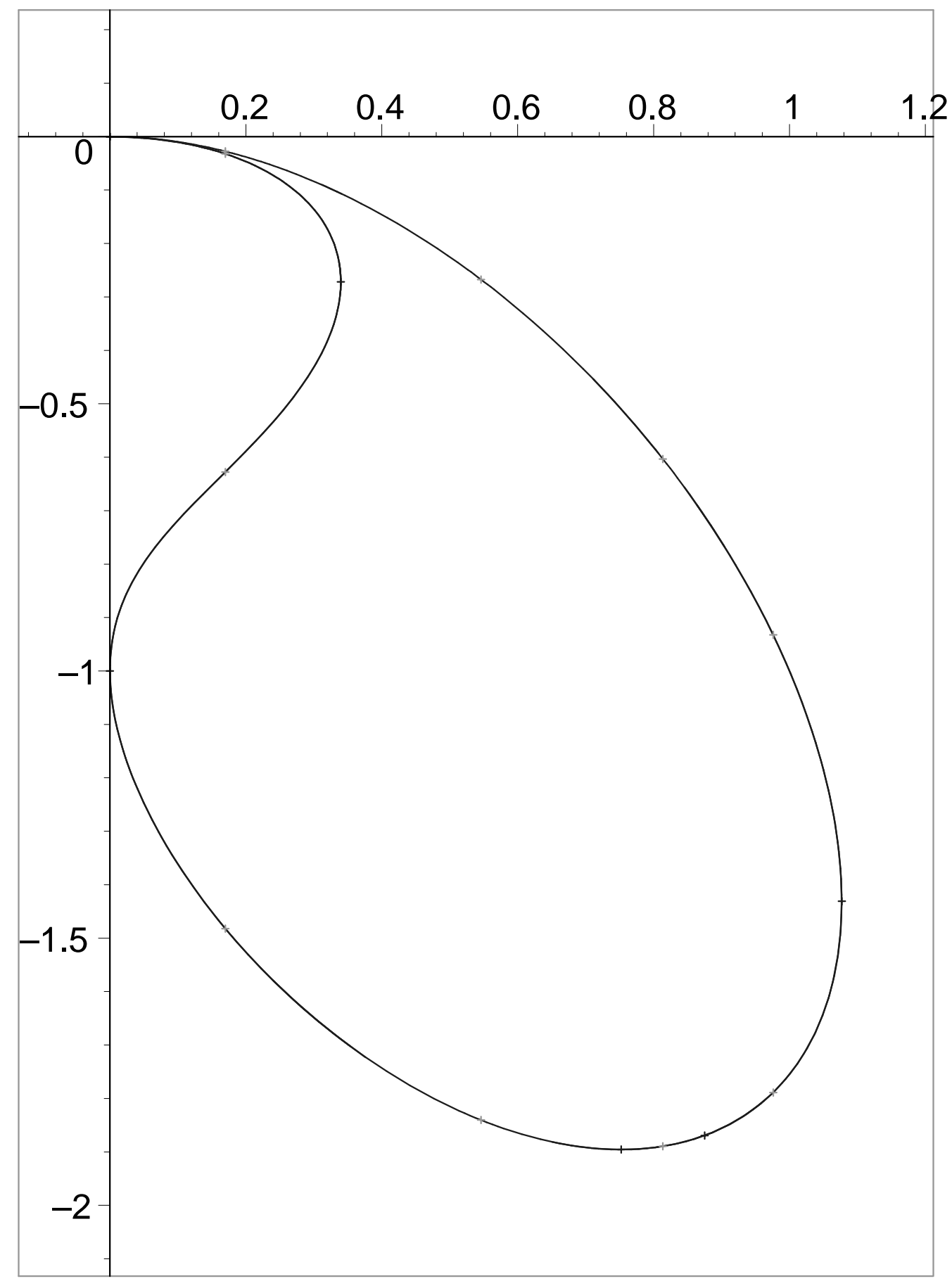




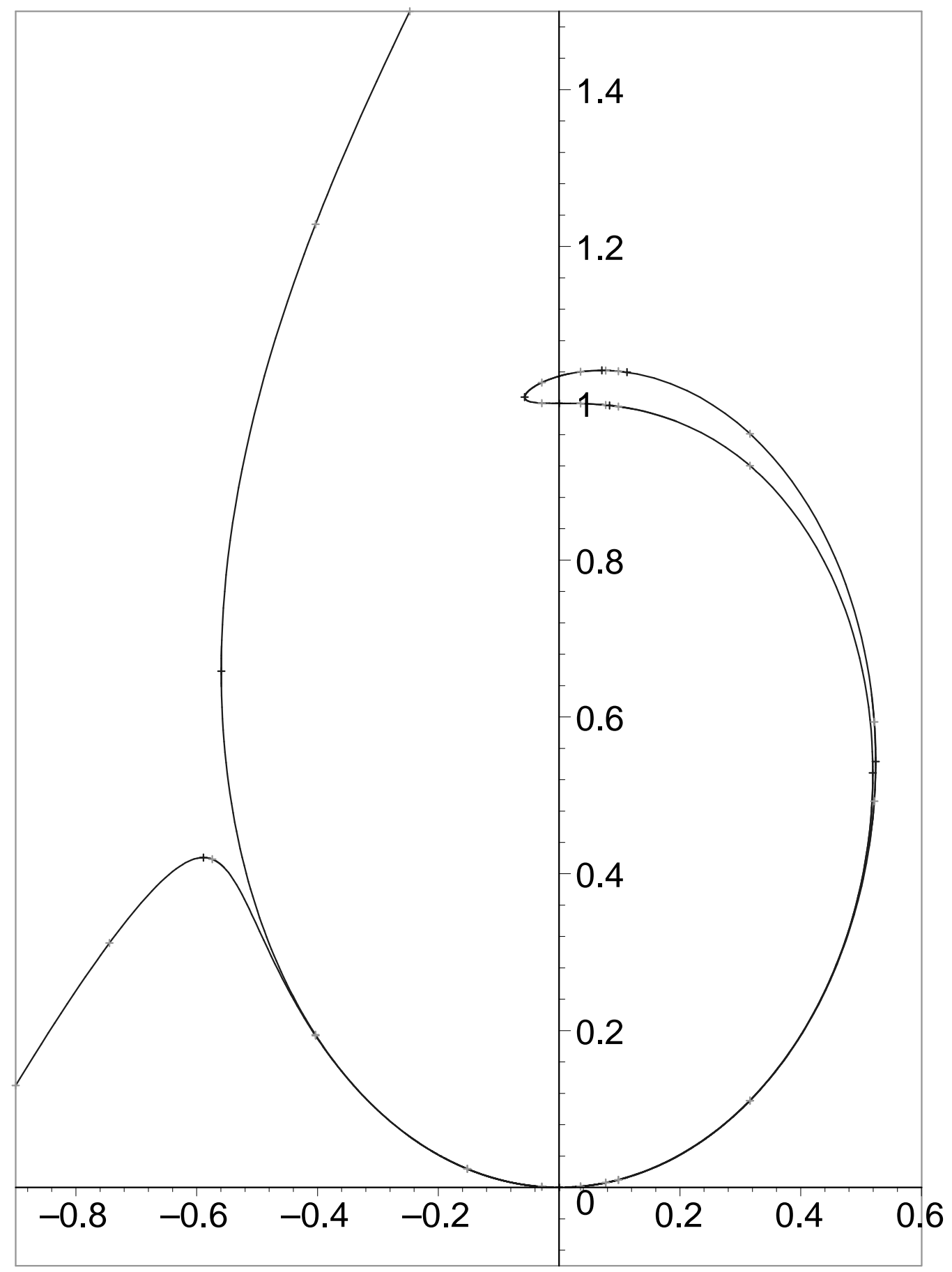




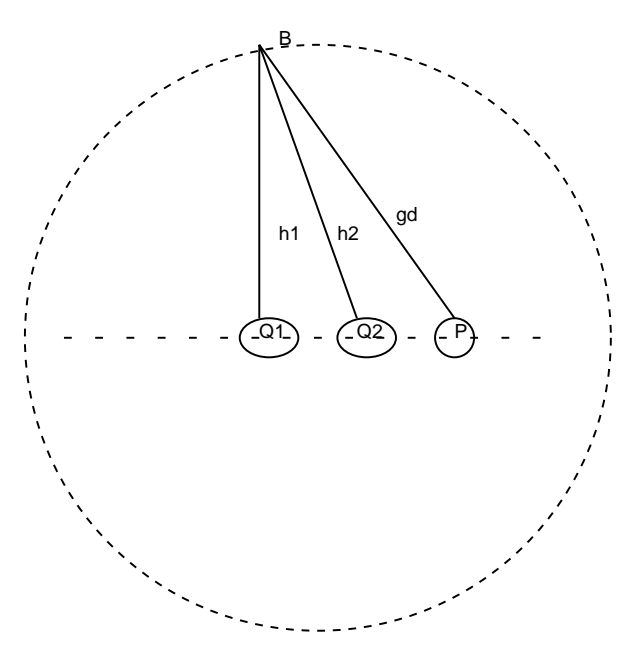




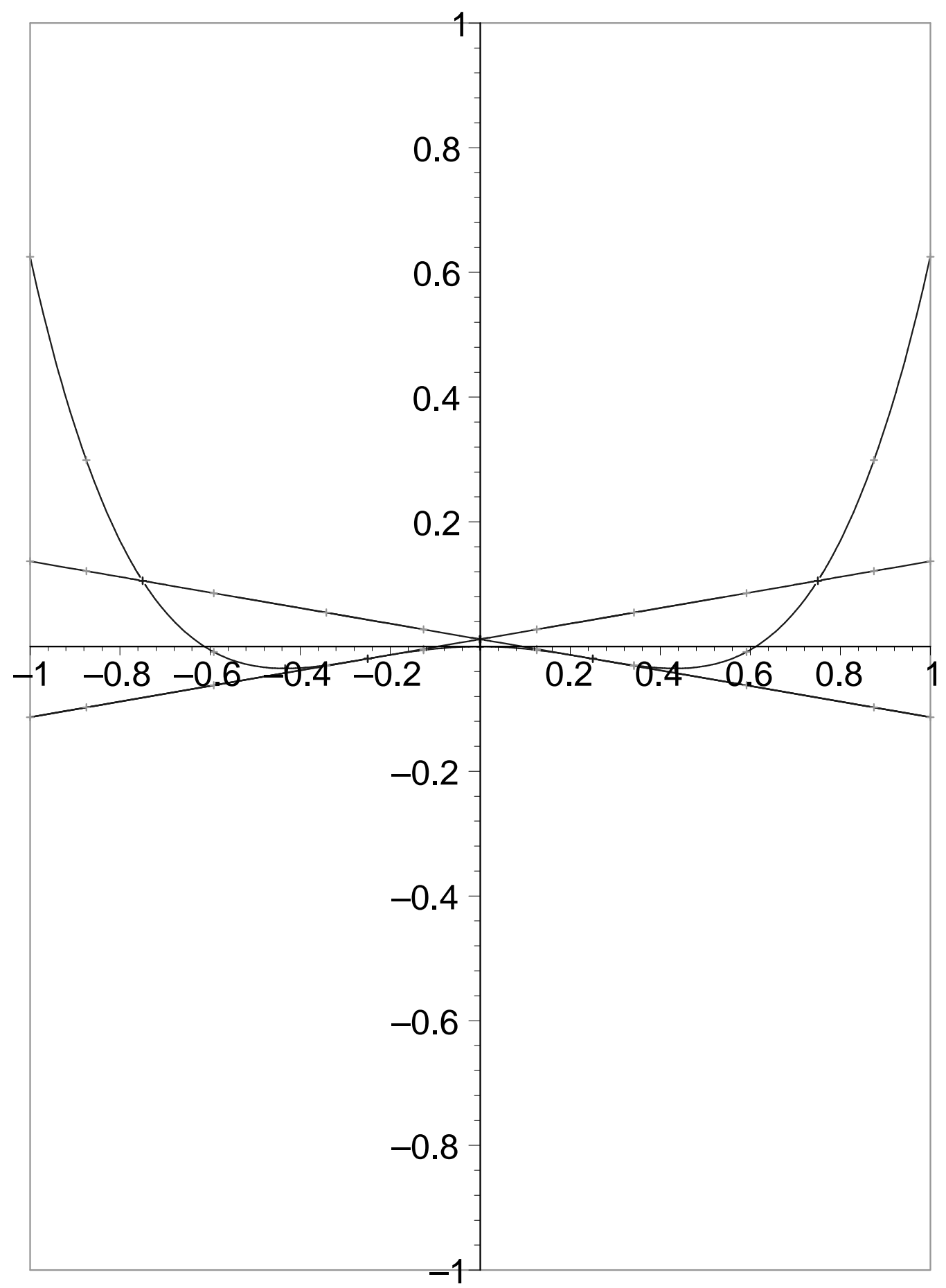

